\title{
Identification of Immunity-Related Genes in Ostrinia furnacalis against Entomopathogenic Fungi by RNA-Seq Analysis
}

\author{
Yang Liu' ${ }^{19}$, Dongxu Shen ${ }^{29}$, Fan Zhou ${ }^{2}$, Guirong Wang ${ }^{1}$, Chunju An ${ }^{2 *}$ \\ 1 State Key Laboratory for Biology of Plant Diseases and Insect Pests, Institute of Plant Protection, Chinese Academy of Agricultural Sciences, Beijing, China, 2 Department \\ of Entomology, College of Agriculture and Biotechnology, China Agricultural University, Beijing, China
}

\begin{abstract}
Background: The Asian corn borer (Ostrinia furnacalis (Guenée)) is one of the most serious corn pests in Asia. Control of this pest with entomopathogenic fungus Beauveria bassiana has been proposed. However, the molecular mechanisms involved in the interactions between $O$. furnacalis and B. bassiana are unclear, especially under the conditions that the genomic information of $O$. furnacalis is currently unavailable. So we sequenced and characterized the transcriptome of $O$. furnacalis larvae infected by $B$. bassiana with special emphasis on immunity-related genes.

Methodology/Principal Findings: Illumina Hiseq2000 was used to sequence 4.64 and $4.72 \mathrm{~Gb}$ of the transcriptome from water-injected and B. bassiana-injected $O$. furnacalis larvae, respectively. De novo assembly generated 62,382 unigenes with mean length of $729 \mathrm{nt}$. All unigenes were searched against $\mathrm{Nt}$, Nr, Swiss-Prot, COG, and KEGG databases for annotations using BLASTN or BLASTX algorithm with an E-value cut-off of $10^{-5}$. A total of $35,700(57.2 \%)$ unigenes were annotated to at least one database. Pairwise comparisons resulted in 13,890 differentially expressed genes, with 5,843 up-regulated and 8,047 down-regulated. Based on sequence similarity to homologs known to participate in immune responses, we totally identified 190 potential immunity-related unigenes. They encode 45 pattern recognition proteins, 33 modulation proteins involved in the prophenoloxidase activation cascade, 46 signal transduction molecules, and 66 immune responsive effectors, respectively. The obtained transcriptome contains putative orthologs for nearly all components of the Toll, Imd, and JAK/STAT pathways. We randomly selected 24 immunity-related unigenes and investigated their expression profiles using quantitative RT-PCR assay. The results revealed variant expression patterns in response to the infection of $B$. bassiana.

Conclusions/Significance: This study provides the comprehensive sequence resource and expression profiles of the immunity-related genes of $O$. furnacalis. The obtained data gives an insight into better understanding the molecular mechanisms of innate immune processes in $O$. furnacalis larvae against $B$. bassiana.
\end{abstract}

Citation: Liu Y, Shen D, Zhou F, Wang G, An C (2014) Identification of Immunity-Related Genes in Ostrinia furnacalis against Entomopathogenic Fungi by RNA-Seq Analysis. PLoS ONE 9(1): e86436. doi:10.1371/journal.pone.0086436

Editor: Xiao-Wei Wang, Zhejiang University, China

Received October 12, 2013; Accepted December 10, 2013; Published January 17, 2014

Copyright: (c) 2014 Liu et al. This is an open-access article distributed under the terms of the Creative Commons Attribution License, which permits unrestricted use, distribution, and reproduction in any medium, provided the original author and source are credited.

Funding: This work was supported by the 973 Program (2013CB114102 and 2013CB127603), National Natural Science Foundation of China (31172090, to C.A. and 31201578, to Y.L.), and Program for New Century Excellent Talents in University (NCET-11-0476, to C.A.). The funders had no role in study design, data collection and analysis, decision to publish, or preparation of the manuscript.

Competing Interests: The authors have declared that no competing interests exist.

*E-mail: anchunju@cau.edu.cn

9 These authors contributed equally to this work.

\section{Introduction}

Innate immunity is an ancient and universal host defense mechanism found in both vertebrates and invertebrates. Insects lack adaptive immunity and mainly rely on innate immunity for defense against the invasion of pathogens or parasites [1,2]. Innate immunity in insects consists of a humoral response based on melanization and antimicrobial peptides (AMPs), and a cellular response including phagocytosis, encapsulation, or nodulation of pathogens [2-6]. Melanization, involving melanin synthesis, sequesters and kills invading microorganisms or parasites $[7,8]$. AMPs are directly cytotoxic to a wide range of microorganims, and are synthesized through the Toll and Imd (Immune deficiency) pathways [9-12].
Gurrent understanding of insect innate immune mechanism is mainly from powerful genetic studies in Drosophila melanogaster $[13,14]$, and biochemical studies in relatively large insects, such as the silkworm, Bombyx mori [15,16], the tobacco hornworm, Manduca sexta [17-19], and the beetle Tenebrio molitor [20,21]. Conceptually, the innate immune reactions in insects can be divided into three steps: (1) recognition of pathogen-associated molecular patterns; (2) signal modulation and transduction; (3) production or replenishment of immune-related molecules including effectors (for review see [22,23]). The recognition step is mediated by a group of proteins, known as pattern recognition proteins (PRPs) such as peptidoglycan recognition protein (PGRP), $\beta$-1,3-glucan recognition protein $(\beta \mathrm{GRP})$ /gram-negative binding protein (GNBP), C-type lectin (CTL) ([24] and references within). A proteolytic cascade composed of a series of serine proteases 
amplifies the initial recognition signal and triggers the activation of signaling pathways $[22,25]$. Ultimately, immune effectors including AMPs are induced in specific tissues, such as fat body (liver analogue) and hemocytes (insect blood cells). These effector molecules combat the invasive microorganisms in the hemolymph (insect blood), and play direct roles in insect innate immunity. So far, knowledge about the identification and involvement of immunity-related genes in various insects, especially in non-model insects, is still unclear and incomplete.

Recently, genome-wide analysis has helped to identify immunity-related genes and gene families in several insect species including 3 dipteran insects D. melanogaster [26], Anopheles gambiae [27], Aedes agypti [28], a hymenopteran insect Apis mellifera [29], a coleopteran insect Tribolium castaneum [30], and a lepidopteran insect B. mori [31]. However, the systematic analysis of immunityrelated genes is hampered in other insects whose genomic information is unavailable. The introduction of novel high through-put sequencing technologies provides insight into a comprehensive immune-gene repertoire of non-model insects. Second generation high-throughput DNA sequencing platforms such as Roche 454, SOLID and Illumina combined with the development of de novo assembly strategies have become powerful tools in transcriptomic studies for non-model organisms without a proper reference genome, and allow targeted identification of genes which are (differentially) expressed upon activation of immune responses [32,33]. This technology has been used, for example, to characterize the immunity-related genes in the beet armyworm Spodoptera exigua [34], the brown planthopper Nilaparvata lugens [35], and the wax moth Galleria mellonella [36] etc. It will greatly facilitate the future studies about analyzing the blueprint of host's genes under microbial challenge, especially for some important insect pests in which molecular information about the immunity-related genes is lacking.

The Asian corn borer, Ostrinia furnacalis (Guenée), is a major insect pest in Asia and causes serious damage on corn, sorghum, millet and cotton [37]. Control of this pest with chemical insecticides is currently hindered by the cryptic nature of larval behavior. Excessive use of chemical insecticides also leads to severe environmental pollution and insecticide residence. Therefore, entomopathogenic fungi become one of promising alternates for its control. The potential for suppression of $O$. furnacalis larvae by entomopathogenic fungus Beauveria bassiana has been proposed [38]. However, the molecular mechanisms involved in the interactions between $O$. furnacalis and $B$. bassiana are still largely unknown, especially under the conditions that the genomic information of $O$. furnacalis is absent currently. This will greatly restrict the further development and wider adoption of entomopathogenic fungi as control agents. The first step to resolve this problem could be comprehensive identification and characterization of immunity-related genes involved in the response of $O$. furnacalis larvae against $B$. bassiana. In this study, we used the Illumina sequencing and de novo assembly to explore the $O$. furnacalis immune response stimulated by $B$. bassiana conidia. We obtained and characterized the transcriptome of $O$. furnacalis larvae with special emphasis on immunity-related genes. 62,382 unigenes were assembled and 35,700 were annotated to known databases. Additionally, we performed quantitative reverse transcript (qRT)PCR analysis to compare the gene expression profiles of $B$. bassiana-infected and non-infected $O$. furnacalis larvae. All these results give us an overview of gene expression profiles of $O$. furnacalis larvae response to B. bassiana, and provide a shortcut for identifying new immunity genes and useful information for studying the molecular basis of host-entomopathogenic fungus interaction.

\section{Materials and Methods}

\section{Insect Rearing}

Asian corn borer (O. furnacalis (Guenée)) was kindly gifted by Dr. Kanglai He from the Institute of Plant Protection, Chinese Academy of Agricultural Sciences. The larvae were reared on an artificial diet at $28^{\circ} \mathrm{C}$ under a relative humidity of $70-90 \%$ and a photoperiod of $16 \mathrm{~h}$ light and $8 \mathrm{~h}$ darkness [39].

\section{B. bassiana Culture and Conidia Suspension Preparation}

B. bassiana strain 252 was cultured on potato dextrose agar (PDA) plates at $25^{\circ} \mathrm{C}$ and $80 \%$ humidity. Conidia (spores) used for infection were harvested from 3-4 weeks old cultures by scraping the surface of the mycelia with sterile cell scrapers into sterile deionized water containing $0.1 \%$ Tween-80. Conidia were separated from other mycelial structures over a sterile funnel packed with autoclaved glass wool, washed twice with $\mathrm{ddH}_{2} \mathrm{O}$ by centrifugation at $4,000 \mathrm{rpm}$, counted and diluted to $2 \times 10^{5}$ conidia/ $\mu$ l. Freshly prepared conidia were used for all experiments.

\section{Immunization and Total RNA Extraction}

Three microliter of diluted conidial suspension $\left(2 \times 10^{5}\right.$ conidia/ $\mu \mathrm{l})$ were injected into the haemocoel of $O$. furnacalis fifth instar day 0 larvae from the same batch. Injection of sterile deionized water was used as a control. After $10 \mathrm{~h}$, each five larvae from challenged or control group were collected, and total RNA samples from the whole body were individually prepared using TRizol Reagent (TIANGEN, Beijing, China) following the manufacturer's instructions. Total RNA was dissolved in $\mathrm{H}_{2} \mathrm{O}$, and RNA quantity was determined on a Nanodrop ND-2000 spectrophotometer (NanoDrop products, Wilmington, DE, USA). RNA integrity was checked on Agilent 2100 BioAnalyzer (Agilent Technologies, Englewood, CO, USA).

\section{Library Construction and Illumina Sequencing}

Ten $\mu \mathrm{g}$ of total RNA equally from 5 larvae in each group was used to isolate mRNA using oligo(dT) magnetic beads. The cDNA library of each sample was constructed using NEBNext ${ }^{\circledR}$ mRNA Library Prep Reagent Set (NEB, Ipswich, MA, USA) following the manufacturer's protocols. Briefly, enriched poly(A) RNA of each sample was fragmented into 200-700 nt pieces with RNA Fragmentation Reagents. The cleaved RNA fragments were transcribed into the first-strand cDNA using random hexamerprimers, followed by second-strand cDNA synthesis. The resulting double-stranded cDNA (dsDNA) was purified with QiaQuick PCR extraction kit (Qiagen, Hilden, Germany) and resolved in EB buffer. The purified dsDNA was treated with T4 DNA Polymerase and T4 Polynucleotide Kinase for end-repairing and dA-tailing. After that, they were ligated to sequencing adaptors with barcode using T4 DNA ligase. Finally, fragments with around 200bplength were purified with QiaQuick GelPurify Kit (Qiagen, Hilden, Germany), and used as templates for PCR amplification to create the cDNA library. The library was paired-end sequenced using PE90 strategy on Illumina HiSeq ${ }^{\text {TM }} 2000$ (Illumina, San Diego, CA, USA) in the Beijing Genome Institute (Shenzhen, China). The challenged and control libraries were sequenced in one lane then raw-reads were sorted out by barcodes.

\section{Assembly and Annotation of Transcriptomes}

Raw reads from each library were filtered to remove low quality reads and the sequence reads containing adapters and poly-A/T tails. The resulting clean reads were assembled to produce unigenes using the short reads assembling program - Trinity 
[40]. The used parameters were as follow: min_glue $=2, \mathrm{~V}=10$, edge-thr $=0.05$, min_kmer_cov $=2$, path_reinforcement_distance $=80$, and group_pairs_distance $=250$. The other parameters were set as the default. Only those contigs with the length no shorter than $48 \mathrm{bp}$ were used for the further assembly. The unigenes from the two samples were pooled together and clustered by TGI Clustering Tool [41]. The following parameters were used to ensure a high quality of assembly: a minimum of $95 \%$ identity, a minimum of 35 overlapping bases, a minimum of 35 scores and a maximum of 25 unmatched overhanging bases at sequence ends. The consensus cluster sequences and singletons make up the final unigene dataset.

For functional annotations, we firstly searched all unigene sequences against various protein databases such as $\mathrm{Nr}$, SwissProt, COG, and KEGG using BLASTX, and then searched nucleotide database Nt using BLASTN, with an E-value cut-off of $10^{-5}$ [42]. The BLAST results were used to extract coding region sequences (CDS) from the unigene sequences. The predicted CDS were further translated into peptide sequences. When a unigene happened to have no BLAST hits, ESTScan software [43] was used to determine the sequence direction. In addition, we performed the Gene Ontology (GO) annotations for each unigene using Blast2GO program, according to the GO associations from the BLASTX against the $\mathrm{Nr}$ database $[44,45]$.

\section{Identification of Differentially Expressed Genes}

A mapping based on expression profile comparison was performed to examine transcript level change between control and treated sample. Clean reads of each library were mapped back to the common non-redundant dataset, respectively, using Bowtie allowing up to 3 base mismatches and a minimum length of $40 \mathrm{bp}$ [46]. The expression of unigene was calculated with FPKM (Fragments per kb per million fragments) method [47]. P-values were calculated according to the hypergeometric test described by Audic and Claverie [48]. The calculated P-values were used to identify differentially expressed genes. The false discovery rate (FDR) was calculated according to the Benjamini and Hochberg algorithm [49], and further used to determine the P-value threshold in multiple testing. Genes with expression changes no less than two folds and FDRs less than 0.001 were considered as significant differentially expressed genes.

\section{Identification and Sequence Analysis of Immunity-related Genes from $O$. furnacalis Transcriptome}

The available immunity-related gene sequences from other insect species were used as references to screen $O$. furnacalis transcriptome database obtained above. The referred insect species mainly included $A$. gambiae, $A$. mellifera, $B$. mori, $D$. melanogaster, M. sexta, T. castaneum, and T. molitor. The potential candidates of $O$. furnacalis immunity-related genes were confirmed by searching the BLASTX algorithm against the non-redundant NCBI nucleotide database using a cut-off E-value of $10^{-5}$.

For the sequence analysis of putative immunity-related genes, the deduced protein domains were determined by using Pfam (http:// www.sanger.ac.uk/Software/Pfam/) and SMART (http://smart. embl.de/). Analysis of deduced amino acid sequences, including prediction of signal peptide, molecular weight and isoelectric point, was carried out in the EXPASY (Expert Protein Analysis System) proteomics server (http://www.expasy.org). Sequence comparisons and phylogenetic analysis were performed by MEGA5 software [50]. Phylogenetic trees were constructed by the neighbor-joining method, with statistical analysis by the bootstrap method, using 1000 repetitions. The phylogenetic trees were visualized in MEGA5 and colored in Adobe Illustrator (Adobe Systems).

\section{Quantitative Reverse Transcript (qRT)-PCR Analysis}

To validate expression profile from comparative transcriptomic analysis, we designed specific primers (Table S1) to perform qRTPCR analysis for 24 immunity-related genes. The annealing temperatures of the primers were controlled at around $62^{\circ} \mathrm{C}$. Total RNA from whole body was extracted independently from 3 biological replicates as described above. DNase I-treated RNA $(1 \mu \mathrm{g})$ was converted into first-strand cDNA using TIANScript RT Kit (TIANGEN, Beijing, China). The cDNA products were diluted 5 fold for use as template. The qRT-PCR was performed on a Applied Biosystems ${ }^{\circledR} 7500$ Real-Time PGR System (Life Technologies, Grand Island, NY, USA) using the GoTaq ${ }^{\circledR}$ qPCR Master (Promega, Madison, WI, USA), according to the manufacturer's instructions. O. furnacalis ribosomal protein L8 $(r p L 8)$ was used as an internal standard to normalize the expression levels. The thermal cycling conditions for qRT-PGR were $95^{\circ} \mathrm{C}$ for $2 \mathrm{~min}$ followed by 40 cycles of $95^{\circ} \mathrm{C}$ for $15 \mathrm{~s}, 55^{\circ} \mathrm{C}$ for $30 \mathrm{~s}$ and $68^{\circ} \mathrm{C}$ for $30 \mathrm{~s}$ ending with a melting curve generation $\left(60^{\circ} \mathrm{C}\right.$ to $95^{\circ} \mathrm{C}$ in increment of $0.5^{\circ} \mathrm{C}$ very $5 \mathrm{~s}$ ). The relative expression of genes was calculated using $2^{-\Delta \Delta \mathrm{Ct}}$ method [51].

\section{Results and Discussion}

\section{Sequencing and Unigene Assembly}

Parasitization of corn borer by B. bassiana provides a good system to study the interactions between insect hosts and entomopathogenic fungi, but the lack of genomic data of corn borer retards the relative progresses. In order to obtain detailed information about corn borer transcriptome, we subjected cDNA from $O$. furnacalis larvae with or without the infection of $B$. bassiana to Hiseq 2000 sequencing. A total of 57,411,104 and 57,669,432 raw reads were generated from water-injected (control) and $B$. bassiana-injected (treated) O. furnacalis libraries, respectively (Table S2). After removal of adaptor sequences, ambiguous reads, and low-quality reads $\left(Q_{2} 2020\right)$, control and treated libraries yielded 51,594,958 (SRA accession number SRX378863) and 52,437,534 (SRA accession number SRX378865) high-quality clean reads comprised of 4,643,546,220 nucleotides (4.64 Gb) and $4,719,378,060$ nucleotides $(4.72 \mathrm{~Gb})$, respectively (Table S2). All high-quality reads were assembled de novo into 95,070 (control) and 96,561 (treated) contigs with a mean length of 371 and $352 \mathrm{nt}$, respectively (Table 1). Using paired-end reads and gap-filling, these contigs were further assembled into 66,004 (mean length 588 nt) and 71,723 (mean length $511 \mathrm{nt}$ ) unigenes (Table 1). These two unigenes-sets were pooled for further clustering, and finally revealed a common non-redundant dataset containing 62,382 unigenes with a mean length of 729 nt, which consist of 22,889 distinct clusters and 39,493 distinct singletons (Table 1). The assembled sequences have been deposited in the NCBI Transcriptome Shotgun Assembly (TSA) Database with the title as BioProject: 228958TSA. The detailed information of each unigene, including gene ID, length, expression and functional annotation, was integrated in Table S3.

\section{Gene Identification, Functional Annotation and Classification}

All 62,382 unigenes were annotated by searching against the databases of Nr, Nt, Swiss-Prot, KEGG, COG, and GO. As shown in Table S4, 31,277 (50.1\%), 18,232 (29.2\%), 22,455 $(36.0 \%), 20,218(32.4 \%), 11,462(18.4 \%)$, and $13,451(21.6 \%)$ unigenes were annotated in the above-referred database, respectively. The rest $(26,682,42.8 \%)$ was not annotated to the existing databases. It suggested that they were the potential sources of novel genes. 
Table 1. Summary for the Illumina sequencing and de novo assembly of $O$. furnacalis transcriptome.

\begin{tabular}{lllllllll}
\hline \multicolumn{1}{l}{} & & & & & & & \\
\hline & Sample & Total number & Total length(nt) & Mean length(nt) & N50 & $\begin{array}{l}\text { Consensus } \\
\text { sequences }\end{array}$ & $\begin{array}{l}\text { Distinct } \\
\text { clusters }\end{array}$ & $\begin{array}{l}\text { Distinct } \\
\text { singletons }\end{array}$ \\
\hline Contig & control & 95,070 & $35,287,785$ & 371 & 706 & - & - & - \\
& treated & 96,561 & $34,000,293$ & 352 & 580 & - & - & - \\
Unigene & control & 66,004 & $38,811,986$ & 588 & 909 & 66,004 & 15,928 & 50,076 \\
All_unigenes & treated & 71,723 & $36,640,515$ & 511 & 757 & 71,723 & 14,576 & 57,147 \\
\hline & & 62,382 & $45,459,381$ & 729 & 1102 & 62,382 & 22,889 & 39,493 \\
\hline
\end{tabular}

doi:10.1371/journal.pone.0086436.t001

The functional annotations of all unigenes were performed mainly based on the BLASTX results against the Nr database. Among the 31,277 annotated unigenes, 15,797 (50.5\%) showed strong homology (E-value smaller than 1e-45), whereas 6101 $(19.5 \%)$ showed poor matches with E-value between 1e-15 and le5 (Figure S1A). The similarity comparison showed 18,299 (58.5\%) unigenes have more than $60 \%$ similarity with known proteins (Figure S1B). For species distribution, 19,139 (61.2\%) annotated unigenes matched to Danaus plexippus, followed by B. mori $(6.4 \%)$, and T. castaneum $(3.8 \%)$ (Figure S1C). Only $246(0.8 \%)$ unigenes matched to the known proteins in European corn borer Ostrinia nubilalis, an closely related specie of $O$. furnacalis (Figure $\mathrm{S1C}$ ). One possible reason was that $O$. nubilalis genome is currently not available in NCBI comparing to the other three insect species mostly matched.

Functional classifications of all unigenes were determined by assigned to Gene Ontology (GO). GO is an international standardized gene functional classification system and covers three categories: biological process, cellular component, and molecular function. In our study, a total of 13,451 unigenes were assigned to one or more GO terms. Among these, 10,146 (75.4\%) unigenes were grouped under the category of biological process, $7,510(55.8 \%)$ under the category of cellular component, and $10,973(81.6 \%)$ under the category of molecular function (Figure $\mathrm{S} 2$ ). The classification of GO terms was further performed at level 2 in each category. As occurred in the transcriptomes of other immune-activated insect larvae [36], the most abundant GO biological process categories were "cellular processes" $(16.0 \%)$ and "metabolic processes" (12.6\%). In this category, 437 unigenes comprise the subcategory of immune system process. In the cellular component category, "cell" (22.3\%) and "cell part" $(22.3 \%)$ represented the most abundant subcategories followed by "organelle" (14.9\%). In the molecular function category, "binding" (41.4\%) and "catalytic activities" (39.5\%) were the most abundant (Figure S2).

We also used COG classifications to analyze the putative protein functions. In total, 11,462 unigenes were functionally classified into 25 COG categories (Figure S3). The cluster for "General function prediction only" (4,333 unigenes) constituted the largest group. The following groups were "Translation, ribosomal structure and biogenesis" (2,388 unigenes), "Replication, recombination and repair" (2,242 unigenes), and "Function unknown" (1,991, 7.3\%) (Figure S3). Only 234 unigenes belonged to the group of "defense mechanisms".

\section{Identification of Differentially Expressed Genes in Response to $B$. bassiana Infection}

To gain insight into the global transcriptional changes taking place in $O$. furnacalis larvae infected by $B$. bassiana conidia, we performed pairwise comparisons between water-injected and $B$. bassiana conidia-injected libraries to identify the differentially expressed genes. The complete lists of differently expressed genes, including their GO and $\mathrm{Nr}$ annotations, are shown in Table S5. The results revealed that 13,890 unigenes exhibited significant changes after $B$. bassiana infection, including 5,843 up-regulated and 8,047 down-regulated unigenes. The detected fold changes (log2ratio) of gene expression ranged from -17.66 to 19.77 .

The GO enrichment was performed to analyze the functions of all identified differently expressed genes. Among the 5,843 upregulated unigenes, 788, 582, and 910 ones were assigned to the categories of biological process, cellular component, and molecular function, respectively. Similarly, 8,047 down-regulated unigenes were also assigned to these three categories: 1,359 in "biological process", 943 in "cellular component", and 1561 in "molecular function" (Figure 1 and Table S5). For both up- and downregulated unigenes, the top 2 most abundant subcategories in each GO category were as follows: "cellular process" and "metabolic process" in "biological process" cluster, "cell" and "cell part" in the "cellular component" cluster, and "binding" and "catalytic activity" in the "molecular function" cluster (Figure 1). In the category of biological process, 42 up-regulated and 58 downregulated genes belonged to the term of immune system process.

To validate the data about differently expressed gene, qRTPCR analysis was performed using specific primers for 24 immunity-related unigenes including 12 up-regulated, 4 downregulated and 8 unchanged unigenes. Data were presented as fold changes normalized to the $r p L 8$ gene in the $B$. bassiana conidiainjected sample relative to the water-injected control sample (Figure 2). Most tested unigenes exhibited the consistent expression trend in qRT-PCR analysis and the original differently expressed genes analysis (Figure 2 and Table S5). It indicates that the results of gene expression profiling from transcriptome analysis are reliable. It is worth noting that the expression profiles of 4 genes including CL1725.Contig1 (OfCTL7), Unigene9842 (OfSpz-1A), unigene13709 (OfToll1), and CL997.Contigl (OfPPO2) were different between qRT-PCR and transcriptome analysis. In the transcriptome analysis, Unigene9842 (OfSpz-1A) and CL997.Contigl (OfPPO2) were slightly decreased while CL1725.Contigl (OfCTL7) and unigene13709 (OfToll1) remained unchanged (Table S5). In the qRT-PCR analysis, all four genes displayed a significant increase in transcript abundance after $B$. bassiana challenge (Figure 2). The observed differences in gene expression might be caused by the difference in the accuracy of these two assay methods. The result from the qRT-PGR analysis could be more accurate because the differently expressed genes identified in this study were from the transcriptome assembly and mapping, but not from the special DGE analysis. 


\section{A}

A Up-regulated, 5843 unigenes

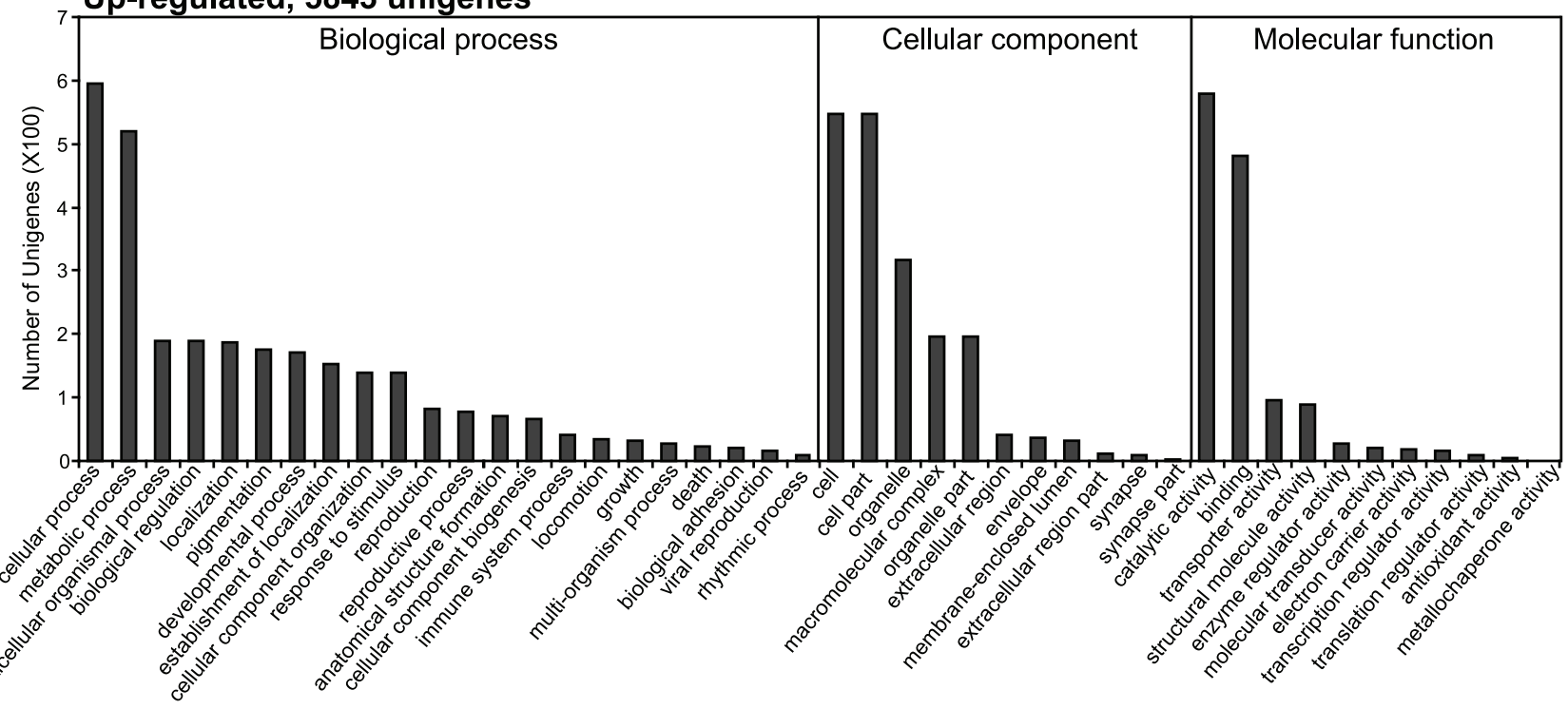

B Down-regulated, 8047 unigenes

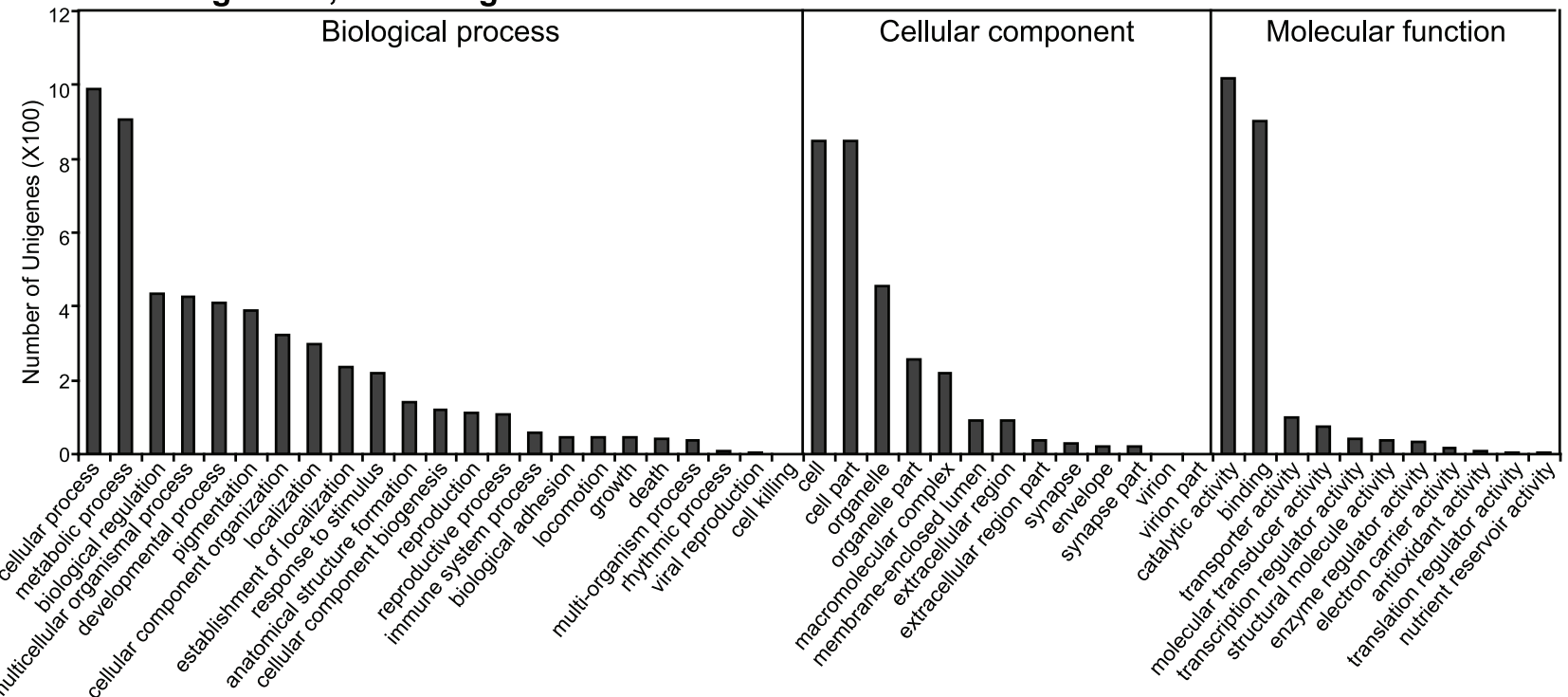

Figure 1. Gene ontology (GO) assignments for the enriched differently expressed unigenes after the infection of $B$. bassiana. (A) 5,843 up-regulated unigenes; (B) 8,047 down-regulated unigenes. The Y-axis shows the number of unigene in each GO term assigned at level 2. doi:10.1371/journal.pone.0086436.g001

\section{Identification of Immunity-related Genes}

Insect innate immune response acts as a crucial part in defending against the infection of pathogens and parasites. In order to obtain the comprehensive perspective on the molecular biology of immune systems in O. furnacalis, we combined GO annotation and BLAST searches to identify the genes related with the cellular and humoral immune response. Based on molecular functions, we have divided the immune-related genes into 4 main groups: genes for signal recognition; genes involved in signal modulation and amplification; genes for signal transduction; and effector genes [52]. In total, we have identified 190 unigenes with high similarity to immunity-related genes, including 45 ones for signal recognition, 33 for signal modulation and amplification, 46 for signal transduction, and 66 for immune effectors (more details seen below). The deduced amino acid sequences of these 190 putative innate immunity genes are listed in Figure S4.

Genes for signal recognition. In insect innate immune response, recognition of nonself is the initial process. It is notably known that the recognition step is mediated by a group of proteins, known as pattern recognition proteins (PRPs), such as peptidoglycan recognition proteins (PGRPs), $\beta$-1,3-glucan recognition protein $(\beta \mathrm{GRPs}) /$ gram-negative binding proteins (GNBPs), Ctype lectins (CTLs), scavenger receptors (SCRs) and so on [24]. In $O$. furnacalis transcriptom, we totally identified at least 45 PRP transcripts including 10 PGRPs, 4 KGRPs, 14 CTLs, 9 SCRs, 2 hemocytins, 1 hemolin, 2 galectins, 1 dscam, 1 draper and 1 eater (Table 2). 

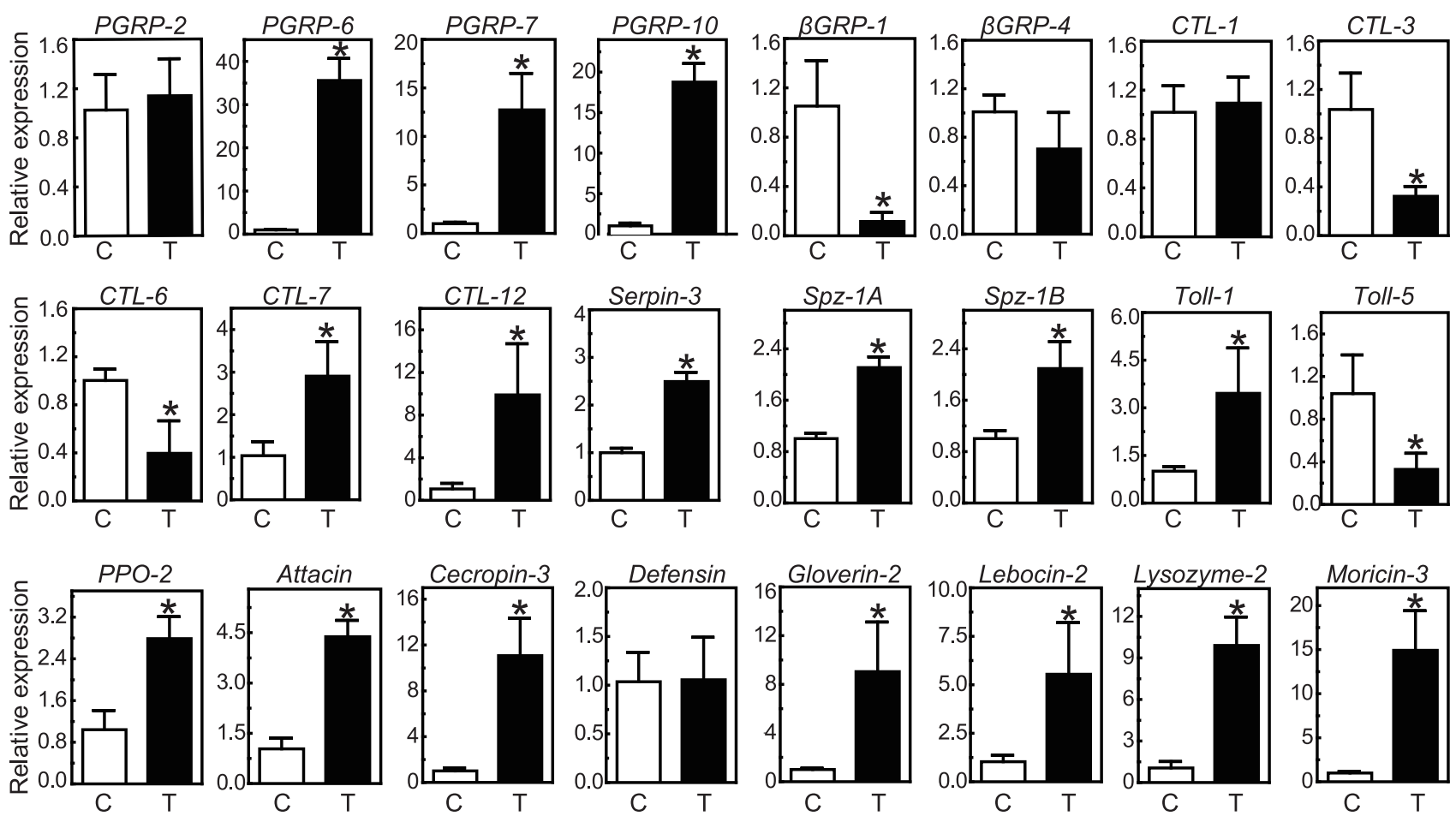

Figure 2. Verification of differentially expressed genes by qRT-PCR. O. furnacalis ribosomal protein L8 ( $r$ L L8) was used as an internal standard to normalize the templates. The bars represent the mean \pm S.D. $(n=3)$. Asterisks indicate means that are significantly different from the control (unpaired t test, $P<0.05$ ). Lack of asterisk indicates the difference is not significant (unpaired $t$ test, $P>0.05$ ).

doi:10.1371/journal.pone.0086436.g002

PGRPs play a central role for the recognition of invading microorganisms in insect immunity by specifically binding to and hydrolyzing bacterial peptidoglycan [53,54]. The first PGRP was isolated from hemolymph of the silkworm, as a pattern recognition receptor to trigger prophenoloxidase (PPO) activation cascade [53]. All PGRP family members share at least one conserved PGRP domain, with similarity to bacteriophage T7 lysozyme, a zinc-dependent N-acetylmuramoyl-L-alanine amidase [55]. The most highly diversified PGRP homologues have been identified in Drosophila. Drosophila has 13 PGRP genes encoding 19 proteins, which are classified into short $(\mathrm{S})$ and long $(\mathrm{L})$ forms $[55,56]$. Among 19 Drosophila PGRPs, six (DmPGRP-SB1/-SB2/$\mathrm{SCla} /-\mathrm{SC} 1 \mathrm{~b} /-\mathrm{SC} 2 /-\mathrm{LB}$ ) have amidase activity, and five (DmPGRP-SA/-SD/-LC/-LE/-LF) lack amidase activity but function as receptors to activate immune signaling pathways [55]. In this study, we identified 10 putative PGRP sequences and designated them as OfPGRP1-10. With the exception of OfPGRP2, the other 9 PGRP transcripts were predicted to be full-lengthed (Table 2). Alignment of 10 putative O. furnacalis PGRPs with Drosophila PGRPs and T7 lysozyme indicated that the deduced amino acid sequences of OfPGRP1-3 and OfPGRP8-10 lack at least one of five active site residues essential for amidase activity in T7 lysozyme (H17, Y46, H122, K128 and C130, K128 is replaced by $\mathrm{T}$ in Drosophila PGRPs). It suggests that these six $O$. furnacalis PGRPs potentially act as receptors for peptidoglycan to initiate a signaling pathway while the left 4 ones (OfPGRP4-7) theoretically have amidase activity and might serve as an intracellular peptidoglycan scavenger. Bootstrap analysis reveals that OfPGRP1 is an ortholog of $M$. sexta PGRP-1 and B. mori PGRP-S1 which both have been verified to function as recognition receptors in the PPO activation cascade [53,54] (Figure 3). It suggests that OfPGRP1 may also act as a peptidoglycan receptor in activating PPO cascade upon the challenge of $B$. bassiana.
Moreover, the analysis of digital expression profile showed that 7 out of 10 identified PGRPs (OfPGRP1, 4-7, 9 and 10) were obviously up-regulated after the infection of $B$. bassiana, whereas the other 3 OfPGRPs remained unchanged (Table 2 and Table S5). We randomly selected 4 PGRP genes (OfPGRP2, 6, 7 and 10) to analyze their transcript changes after the injection of $B$. bassiana using qRT-PCR methods. OfPGRP-6, -7 and -10 showed very high expression levels in $B$. bassiana-injected corn borer larvae, while OfPGRP2 mRNA level was consistent (Figure 2). We speculated that $O$. furnacalis PGRPs might play different roles, and work in concert with each other to defend against the invasion of $B$. bassiana.

$\beta G R P / G N B P$ belongs to another pattern recognition protein family. This family contains two functionally different proteins, one of which has a strong affinity to $\beta$-1,3-glucans of fungal cell walls ( $\beta$ GRP) and the other is dubbed as Gram-negative binding protein (GNBP) but binds to Gram-negative bacteria or Grampositive bacteria [57]. Since the first $\beta$ GRP was identified in the PPO-activation system of B. mori [58], $\beta$ GRPs have been identified in insects including Drosophila (3 genes) [57], Anopheles (7 genes) [27], Apis (2 genes) [29], Manduca (2 genes) [59,60], and Tribolium (3 genes) [30]. They all consist of a conserved N-terminal $\beta-1,3-$ glucan-recognition domain for the detection of pathogens or parasites, and a C-terminal glucanase-like domain with undefined function $[61,62]$. Three-dimensional structures of Bm $\beta$ GRP1 and DmGNBP3 further revealed that the $\mathrm{N}$-terminal $\beta$-1,3-glucanrecognition domain actually adopts a $\beta$-sandwich structure formed by eight $\beta$-strands $[62,63]$. In this study, we identified $4 \beta G R P /$ $G N B P$ genes with predicted full length, and designated them as OfBGRP1-4. All Of $\beta$ GRPs are assumed to be secreted proteins because they have putative signal peptides (Table 2). A comparison of the deduced amino acid sequences with Drosophila GNBP13 and Bombyx $\beta$ GRP1 showed that Of $\beta$ GRP1-3 contained the 
Table 2. Summary of the putative immunity-related unigenes identified in $O$. furnacalis transcriptome.

\begin{tabular}{|c|c|c|c|c|c|c|c|c|}
\hline Designated name & Unigene ID & $\begin{array}{l}\text { Nucleotide } \\
\text { length (nt) }\end{array}$ & $\begin{array}{l}\text { Full } \\
\text { length }^{a}\end{array}$ & $\begin{array}{l}\text { Protein } \\
\text { length }(\mathrm{aa})^{\mathrm{b}}\end{array}$ & $\begin{array}{l}\text { Predicted } \\
\text { signal peptide }^{c}\end{array}$ & $\begin{array}{l}\text { Fold } \\
\text { change }^{d}\end{array}$ & P value & $\begin{array}{l}\text { Identity to } \\
\text { best hit (\%) }\end{array}$ \\
\hline \multicolumn{9}{|l|}{ Recognition } \\
\hline \multicolumn{9}{|l|}{ PGRP } \\
\hline OfPGRP1 & CL244.Contig2 & 2759 & Yes & 194 & $1-20 G * E$ & 3.5037 & 0 & 53 \\
\hline OfPGRP2 & CL3377.Contig2 & 969 & $3^{\prime}$ & 286 & $\mathrm{~N} / \mathrm{A}$ & 0.2783 & 0.58704 & 65 \\
\hline OfPGRP3 & CL6326.Contig1 & 1423 & Yes & 255 & & 0.0976 & 0.73835 & 32 \\
\hline OfPGRP4 & Unigene16261 & 1356 & Yes & 238 & $1-18 G^{*} \mathrm{H}$ & 7.3068 & 0 & 97 \\
\hline OfPGRP5 & CL7407.Contig1 & 1638 & Yes & 231 & $1-18 G^{*} \mathrm{H}$ & 6.7338 & 0 & 100 \\
\hline OfPGRP6 & Unigene20173 & 1042 & Yes & 211 & $1-19 \mathrm{~T}^{*} \mathrm{H}$ & 6.0695 & 0 & 97 \\
\hline OfPGRP7 & Unigene287 & 1100 & Yes & 206 & $1-19 G * F$ & 4.4392 & 0 & 58 \\
\hline OfPGRP8 & Unigene2928 & 1927 & Yes & 299 & & -1.0262 & $4.79 \mathrm{E}-22$ & 53 \\
\hline OfPGRP9 & Unigene6285 & 642 & Yes & 190 & $1-18 S^{*} \mathrm{~L}$ & 1.5042 & $1.25 \mathrm{E}-241$ & 98 \\
\hline OfPGRP10 & Unigene10881 & 732 & Yes & 181 & $1-17 T^{*} \mathrm{~L}$ & 5.0527 & 0 & 55 \\
\hline \multicolumn{9}{|l|}{$\beta G R P$} \\
\hline Of $\beta$ GRP1 & Unigene4875 & 1684 & Yes & 490 & $1-17 A^{*} Q$ & -0.5675 & $3.39 \mathrm{E}-115$ & 67 \\
\hline Of $\beta G R P 2$ & CL5474.Contig2 & 1986 & Yes & 490 & $1-25 S^{*} \mathrm{~V}$ & 1.2107 & 0 & 61 \\
\hline Of $\beta$ GRP3 & Unigene9808 & 1717 & Yes & 491 & $1-20 C^{*} R$ & -0.2678 & $9.813 \mathrm{E}-44$ & 66 \\
\hline Of $\beta$ GRP4 & Unigene6628 & 1445 & Yes & 375 & $1-17 A^{*} C$ & 1.8395 & 0 & 99 \\
\hline \multicolumn{9}{|l|}{ C-type lectin } \\
\hline OfCTL1 & CL106.Contig3 & 1408 & Yes & 328 & $1-21 T^{*} \mathrm{D}$ & 0.2613 & $1.903 \mathrm{E}-12$ & 51 \\
\hline OfCTL2 & Unigene5701 & 1211 & Yes & 322 & & 0.3521 & $8.172 \mathrm{E}-05$ & 52 \\
\hline OfCTL3 & Unigene5307 & 1695 & Yes & 321 & $1-21 S^{*} Q$ & -1.3973 & 0 & 62 \\
\hline OfCTL4 & Unigene2023 & 1133 & Yes & 321 & $1-17 G * S$ & 0.626 & $3.885 \mathrm{E}-58$ & 39 \\
\hline OfCTL5 & Unigene2411 & 1583 & Yes & 319 & & -2.5674 & 0 & 61 \\
\hline OfCTL6 & Unigene14484 & 1034 & Yes & 314 & $1-22 G^{*} R$ & -0.2353 & $5.40 \mathrm{E}-87$ & 62 \\
\hline OfCTL7 & CL1725.Contig1 & 1197 & Yes & 307 & $1-20 S^{*} S$ & -1.3936 & $5.94 \mathrm{E}-26$ & 99 \\
\hline OfCTL8 & Unigene22572 & 1034 & Yes & 301 & $1-19 A^{*} Q$ & -0.2829 & $1.31 \mathrm{E}-05$ & 47 \\
\hline OfCTL9 & Unigene31526 & 1094 & $3^{\prime}$ & 300 & $\mathrm{~N} / \mathrm{A}$ & -0.415 & $5.57 \mathrm{E}-02$ & 50 \\
\hline OfCTL10 & CL8286.Contig1 & 1072 & $3^{\prime}$ & 250 & $\mathrm{~N} / \mathrm{A}$ & -2.2983 & $1.63 \mathrm{E}-13$ & 94 \\
\hline OfCTL11 & Unigene6545 & 1102 & Yes & 223 & $1-19 A^{*} Q$ & -1.5713 & $3.24 \mathrm{E}-204$ & 92 \\
\hline OfCTL12 & CL4786.Contig1 & 1540 & Yes & 220 & $1-19 A^{*} Q$ & 3.8157 & 0 & 94 \\
\hline OfCTL13 & Unigene9847 & 1743 & Yes & 207 & $1-21 A^{*} Q$ & -3.5946 & 0 & 87 \\
\hline OfCTL14 & CL321.Contig3 & 763 & Yes & 184 & $1-21 T^{*} \mathrm{~V}$ & -0.1434 & 0.68032 & 88 \\
\hline \multicolumn{9}{|l|}{ galectin } \\
\hline OfGalectin1 & CL1314.Contig2 & 1433 & Yes & 365 & & 0.1771 & $3.65 \mathrm{E}-01$ & 67 \\
\hline OfGalectin2 & Unigene31759 & 1011 & Yes & 255 & & 1.9018 & 5.87E-96 & 41 \\
\hline \multicolumn{9}{|c|}{ scavenger receptor (SCR) } \\
\hline OfSCR1 & CL3884.Contig1 & 2733 & Yes & 737 & & 0.3777 & 0.039813 & 56 \\
\hline OfSCR2 & Unigene3524 & 2348 & Yes & 626 & & -0.6523 & $2.98 \mathrm{E}-09$ & 75 \\
\hline OfSCR3 & Unigene4658 & 2326 & Yes & 566 & & 0.1914 & $1.47 \mathrm{E}-02$ & 60 \\
\hline OfSCR4 & Unigene12877 & 1689 & $5^{\prime}$ & 521 & & -0.2322 & $5.40 \mathrm{E}-05$ & 33 \\
\hline OfSCR5 & CL2453.Contig1 & 2411 & Yes & 515 & & -0.031 & 7.61E-01 & 89 \\
\hline OfSCR6 & Unigene18387 & 1692 & $5^{\prime}$ & 511 & & -2.9157 & $8.78 \mathrm{E}-36$ & 41 \\
\hline OfSCR7 & CL675.Contig2 & 2033 & Yes & 496 & & 0.3464 & $2.34 \mathrm{E}-07$ & 67 \\
\hline OfSCR8 & Unigene3062 & 1832 & Yes & 439 & $1-16 A^{*} Q$ & -2.9802 & 0 & 53 \\
\hline OfSCR9 & Unigene3589 & 1450 & $3^{\prime}$ & 425 & N/A & -4.8973 & $3.78 \mathrm{E}-69$ & 36 \\
\hline \multicolumn{9}{|l|}{ hemocytin } \\
\hline OfHemocytin 1 & Unigene72 & 4646 & $3^{\prime}$ & 1497 & $\mathrm{~N} / \mathrm{A}$ & -3.2442 & 0 & 61 \\
\hline OfHemocytin2 & Unigene2798 & 2134 & $M$ & 710 & $\mathrm{~N} / \mathrm{A}$ & -2.8968 & $2.45 \mathrm{E}-41$ & 43 \\
\hline
\end{tabular}


Table 2. Cont.

\begin{tabular}{|c|c|c|c|c|c|c|c|c|}
\hline Designated name & Unigene ID & $\begin{array}{l}\text { Nucleotide } \\
\text { length (nt) }\end{array}$ & $\begin{array}{l}\text { Full } \\
\text { length }^{\mathrm{a}}\end{array}$ & $\begin{array}{l}\text { Protein } \\
{\text { length }(a a)^{b}}^{b}\end{array}$ & $\begin{array}{l}\text { Predicted } \\
\text { signal peptide }^{c}\end{array}$ & $\begin{array}{l}\text { Fold } \\
\text { change }\end{array}$ & P value ${ }^{e}$ & $\begin{array}{l}\text { Identity to } \\
\text { best hit (\%) }\end{array}$ \\
\hline \multicolumn{9}{|l|}{ hemolin } \\
\hline OfHemolin & Unigene9924 & 1649 & Yes & 456 & & 2.7711 & 0 & 47 \\
\hline \multicolumn{9}{|l|}{ dscam } \\
\hline OfDscam & CL3756.Contig3 & 3280 & M & 1093 & $\mathrm{~N} / \mathrm{A}$ & -1.4098 & $5.39 \mathrm{E}-44$ & 85 \\
\hline \multicolumn{9}{|l|}{ Draper } \\
\hline OfDraper & Unigene5316 & 3199 & $5^{\prime}$ & 954 & $1-17 A^{*} \mathrm{~L}$ & -0.5145 & $6.75 \mathrm{E}-21$ & 53 \\
\hline \multicolumn{9}{|l|}{ Eater } \\
\hline OfEater & Unigene6859 & 2266 & Yes & 664 & $1-19 S * D$ & -1.5664 & $2.19 \mathrm{E}-35$ & 41 \\
\hline \multicolumn{9}{|l|}{ Modulation } \\
\hline \multicolumn{9}{|c|}{ Clip-domain serine protease } \\
\hline OfSP1 & CL399.Contig2 & 1992 & Yes & 358 & $1-20 A^{*} Q$ & -0.7183 & $2.12 \mathrm{E}-08$ & 56 \\
\hline OfSP2 & Unigene18713 & 1454 & Yes & 421 & $1-18 G * A$ & 0.2364 & $5.82 \mathrm{E}-19$ & 57 \\
\hline OfSP3 & CL5234.Contig1 & 1602 & Yes & 400 & $1-21 A^{*} Q$ & -0.8512 & $1.3 \mathrm{E}-10$ & 54 \\
\hline OfSP4 & Unigene13481 & 1579 & Yes & 444 & $1-17 S * S$ & 0.2868 & $5.13 \mathrm{E}-06$ & 67 \\
\hline OfSP5 & CL1110.Contig5 & 1381 & Yes & 385 & $1-17 A^{*} E$ & -0.6014 & $3.69 \mathrm{E}-03$ & 34 \\
\hline OfSP7 & CL2452.Contig1 & 1865 & Yes & 421 & $1-19 G * Q$ & 1.7753 & 0 & 44 \\
\hline OfSP8 & CL4641.Contig3 & 2105 & Yes & 424 & $1-19 G * Q$ & 1.4516 & 0 & 63 \\
\hline OfSP10 & Unigene941 & 1669 & Yes & 400 & $1-18 A^{*} N$ & -1.0384 & $4.13 E-297$ & 99 \\
\hline OfSP12 & Unigene3077 & 1873 & Yes & 370 & $1-21 A^{*} Q$ & 1.0886 & 0 & 64 \\
\hline OfSP13 & Unigene17162 & 1338 & Yes & 380 & $1-19 T^{*} Q$ & 1.2121 & $1.56 \mathrm{E}-296$ & 67 \\
\hline OfSP14 & Unigene21915 & 1830 & Yes & 451 & & 0.1695 & $1.01 \mathrm{E}-08$ & 57 \\
\hline OfSP17 & Unigene21394 & 2158 & Yes & 530 & $1-26 S^{*} \mathrm{E}$ & -2.6323 & 0 & 55 \\
\hline OfSP37 & CL5321.Contig1 & 1380 & Yes & 389 & $1-16 G * D$ & -2.3145 & $1.40 \mathrm{E}-101$ & 70 \\
\hline \multicolumn{9}{|c|}{ Clip-domain serine protease homolog (SPH) } \\
\hline OfSPH8 & Unigene10046 & 1469 & Yes & 411 & $1-15 A^{*} Q$ & 0.6276 & $2.22 \mathrm{E}-196$ & 62 \\
\hline OfSPH9 & CL945.Contig1 & 2031 & Yes & 604 & $1-17 A^{*} D$ & -2.2505 & $1.77 \mathrm{E}-203$ & 43 \\
\hline OfSPH10 & Unigene 12563 & 1321 & Yes & 399 & $1-20 G * Q$ & -1.2335 & 0 & 36 \\
\hline \multicolumn{9}{|l|}{ serpin } \\
\hline OfSerpin-1A & CL2978.Contig1 & 1497 & Yes & 397 & $1-16 A^{*} D$ & -0.0006 & 9.95E-01 & 58 \\
\hline OfSerpin-1B & CL2978.Contig2 & 1564 & Yes & 406 & $1-16 A^{*} D$ & -0.097 & $1.35 \mathrm{E}-01$ & 59 \\
\hline OfSerpin-1C & CL2978.Contig3 & 1450 & Yes & 393 & $1-16 A^{*} D$ & -1.3272 & 2.89E-235 & 58 \\
\hline OfSerpin-1D & Unigene18304 & 2181 & Yes & 395 & $1-16 A^{*} D$ & -0.0419 & 2.92E-01 & 62 \\
\hline OfSerpin-2 & CL2637.Contig5 & 1544 & Yes & 377 & & 0.9159 & $5.328 \mathrm{E}-16$ & 69 \\
\hline OfSerpin-3 & CL7904.Contig2 & 1770 & $3^{\prime}$ & 375 & $\mathrm{~N} / \mathrm{A}$ & 2.3578 & 0 & 68 \\
\hline OfSerpin-4 & CL9195.Contig5 & 1561 & Yes & 413 & $1-18 G * Q$ & 4.7853 & 0 & 63 \\
\hline OfSerpin-5 & CL5373.Contig1 & 1478 & Yes & 395 & $1-16 C^{*} A$ & -1.2879 & $3.16 E-219$ & 72 \\
\hline OfSerpin-6 & CL5354.Contig1 & 1570 & $3^{\prime}$ & 295 & $\mathrm{~N} / \mathrm{A}$ & 1.6927 & $1.24 \mathrm{E}-163$ & 88 \\
\hline OfSerpin-7 & CL3593.Contig1 & 2276 & Yes & 719 & $1-21 A^{*} G$ & -2.9453 & 0 & 55 \\
\hline OfSerpin-8 & CL60.Contig2 & 2034 & Yes & 646 & $1-20 A^{*} D$ & -2.6132 & $4.20 \mathrm{E}-60$ & 39 \\
\hline OfSerpin-9 & CL3162.Contig2 & 2540 & Yes & 542 & $1-18 A^{*} Q$ & 0.0382 & $4.08 \mathrm{E}-02$ & 55 \\
\hline OfSerpin-10 & Unigene7142 & 1880 & Yes & 518 & $1-17 A^{*} R$ & -2.0645 & $3.48 \mathrm{E}-118$ & 73 \\
\hline OfSerpin-11 & CL2102.Contig3 & 2110 & Yes & 457 & $1-19 P * R$ & -0.3346 & $5.16 \mathrm{E}-06$ & 63 \\
\hline OfSerpin-12 & Unigene868 & 1592 & Yes & 428 & $1-23 G * G$ & 1.0433 & $3.26 \mathrm{E}-124$ & 68 \\
\hline OfSerpin-13 & Unigene 17483 & 1377 & Yes & 406 & $1-20 G * Q$ & -0.5347 & $6.74 \mathrm{E}-37$ & 51 \\
\hline OfSerpin-14 & Unigene9805 & 1011 & $3^{\prime}$ & 296 & $\mathrm{~N} / \mathrm{A}$ & 0.8088 & $1.88 \mathrm{E}-06$ & 48 \\
\hline \multicolumn{9}{|l|}{ Transduction } \\
\hline \multicolumn{9}{|l|}{ (Toll pathway) } \\
\hline \multicolumn{9}{|l|}{ Spätzle } \\
\hline OfSpz1A & Unigene 9842 & 1126 & Yes & 319 & $1-17 A^{*} Y$ & -0.367 & $3.26 \mathrm{E}-03$ & 33 \\
\hline
\end{tabular}


Table 2. Cont.

\begin{tabular}{|c|c|c|c|c|c|c|c|c|}
\hline Designated name & Unigene ID & $\begin{array}{l}\text { Nucleotide } \\
\text { length (nt) }\end{array}$ & $\begin{array}{l}\text { Full } \\
\text { length }\end{array}$ & $\begin{array}{l}\text { Protein } \\
\text { length }(a a)^{b}\end{array}$ & $\begin{array}{l}\text { Predicted } \\
\text { signal peptide }^{c}\end{array}$ & $\begin{array}{l}\text { Fold } \\
\text { change }^{d}\end{array}$ & P value ${ }^{e}$ & $\begin{array}{l}\text { Identity to } \\
\text { best hit }(\%)^{f}\end{array}$ \\
\hline OfSpz1B & Unigene6173 & 773 & Yes & 221 & $1-18 A^{*} Y$ & -0.0271 & $8.16 \mathrm{E}-01$ & 32 \\
\hline OfSpz3 & Unigene6845 & 1539 & $3^{\prime}$ & 481 & $\mathrm{~N} / \mathrm{A}$ & -0.308 & $1.58 \mathrm{E}-01$ & 73 \\
\hline OfSpz4 & Unigene18729 & 1291 & $5^{\prime}$ & 413 & $1-17 A^{*} Y$ & 2.1258 & $5.77 \mathrm{E}-20$ & 62 \\
\hline OfSpz5 & Unigene27639 & 1584 & Yes & 418 & $1-28 G^{*} Y$ & -3.3208 & $3.13 \mathrm{E}-26$ & 33 \\
\hline OfSpz6 & Unigene7136 & 1322 & $3^{\prime}$ & 398 & N/A & 1.9615 & $3.19 E-11$ & 56 \\
\hline \multicolumn{9}{|l|}{ Toll } \\
\hline OfToll1 & Unigene13709 & 4736 & Yes & 1340 & $1-22 G^{*} A$ & -0.227 & $5.67 \mathrm{E}-04$ & 89 \\
\hline OfToll2 & CL3792.Contig1 & 3426 & Yes & 1003 & $1-22 A^{*} C$ & -1.1004 & $7.55 \mathrm{E}-89$ & 32 \\
\hline OfToll3 & Unigene7123 & 2672 & Yes & 770 & $1-22 C^{*} S$ & -0.4329 & $8.24 \mathrm{E}-06$ & 36 \\
\hline OfToll4 & CL1140.Contig1 & 2841 & Yes & 745 & $1-19 G * F$ & -0.0957 & $1.70 \mathrm{E}-01$ & 51 \\
\hline OfToll5 & Unigene9148 & 2622 & $3^{\prime}$ & 737 & $\mathrm{~N} / \mathrm{A}$ & -2.2303 & $6.64 \mathrm{E}-57$ & 79 \\
\hline OfToll6 & CL8435.Contig1 & 2420 & $3^{\prime}$ & 709 & N/A & -0.5819 & $3.94 \mathrm{E}-04$ & 53 \\
\hline OfToll7 & Unigene150 & 1832 & $3^{\prime}$ & 588 & $\mathrm{~N} / \mathrm{A}$ & -1.69 & $3.61 \mathrm{E}-13$ & 95 \\
\hline OfToll8 & Unigene16267 & 1800 & Yes & 425 & $1-19 G^{*} E$ & -0.993 & $4.92 \mathrm{E}-26$ & 29 \\
\hline OfToll9 & Unigene16850 & 1350 & $3^{\prime}$ & 417 & N/A & -1.0493 & $7.53 \mathrm{E}-84$ & 40 \\
\hline OfToll10 & Unigene5256 & 1227 & M & 409 & $\mathrm{~N} / \mathrm{A}$ & -0.3618 & $1.05 \mathrm{E}-01$ & 87 \\
\hline OfToll11 & Unigene2699 & 1022 & $3^{\prime}$ & 320 & $\mathrm{~N} / \mathrm{A}$ & 0.0403 & $8.61 \mathrm{E}-01$ & 84 \\
\hline OfToll12 & Unigene13736 & 917 & $M$ & 305 & N/A & -1.9247 & 1.19E-07 & 80 \\
\hline \multicolumn{9}{|l|}{ MyD88 } \\
\hline OfMyD88-1 & Unigene142 & 2405 & Yes & 379 & & -0.2001 & $4.00 \mathrm{E}-02$ & 42 \\
\hline OfMyD88-2 & CL1575.Contig1 & 1567 & Yes & 378 & & 0.5748 & $1.73 \mathrm{E}-13$ & 59 \\
\hline \multicolumn{9}{|l|}{ Tube } \\
\hline OfTube & Unigene3838 & 427 & 5 & 94 & & -0.6087 & $1.15 \mathrm{E}-02$ & 39 \\
\hline \multicolumn{9}{|l|}{ Pelle } \\
\hline OfPelle1 & Unigene9421 & 2053 & $3^{\prime}$ & 507 & $\mathrm{~N} / \mathrm{A}$ & -0.6058 & $1.14 \mathrm{E}-09$ & 29 \\
\hline OfPelle2 & CL1687.Contig1 & 2390 & Yes & 483 & & 0.6875 & $1.27 \mathrm{E}-16$ & 40 \\
\hline \multicolumn{9}{|l|}{ Pellino } \\
\hline OfPellino & CL6004.Contig1 & 2131 & Yes & 432 & & -0.0473 & $5.99 \mathrm{E}-01$ & 74 \\
\hline \multicolumn{9}{|l|}{ TRAF2 } \\
\hline OfTRAF2 & Unigene19082 & 1146 & $5^{\prime}$ & 358 & & -0.7026 & $2.76 \mathrm{E}-03$ & 79 \\
\hline \multicolumn{9}{|l|}{ Cactus } \\
\hline OfCactus & CL1201.Contig1 & 3009 & Yes & 335 & & 3.0653 & 0 & 48 \\
\hline \multicolumn{9}{|l|}{ Dorsal/Dif } \\
\hline OfDorsal & CL7451.Contig1 & 2888 & Yes & 615 & & 0.2955 & $2.26 \mathrm{E}-03$ & 50 \\
\hline \multicolumn{9}{|l|}{ Tollip } \\
\hline OfTollip & CL951.Contig1 & 1116 & Yes & 276 & & -0.3531 & $1.23 \mathrm{E}-02$ & 62 \\
\hline \multicolumn{9}{|l|}{ (Imd pathway) } \\
\hline \multicolumn{9}{|l|}{ Imd } \\
\hline Oflmd & CL2356.Contig1 & 1122 & Yes & 253 & & 0.4303 & $9.28 \mathrm{E}-07$ & 48 \\
\hline \multicolumn{9}{|l|}{ Dredd } \\
\hline OfDredd & CL978.Contig1 & 1474 & $5^{\prime}$ & 427 & & 0.1654 & $2.92 \mathrm{E}-02$ & 42 \\
\hline \multicolumn{9}{|l|}{ FADD } \\
\hline OfFADD & Unigene6623 & 860 & Yes & 221 & & -0.0392 & $5.36 \mathrm{E}-01$ & 45 \\
\hline \multicolumn{9}{|l|}{ relish } \\
\hline OfRelish & CL6686.Contig2 & 3768 & Yes & 956 & & 1.916 & 0 & 52 \\
\hline \multicolumn{9}{|l|}{ caspar } \\
\hline OfCaspar & CL1175.Contig1 & 2264 & $3^{\prime}$ & 665 & $\mathrm{~N} / \mathrm{A}$ & -0.5822 & 5.67E-06 & 41 \\
\hline \multicolumn{9}{|l|}{ IKK } \\
\hline OfIKK $\gamma$ & Unigene2344 & 3484 & Yes & 681 & & 2.742 & 0 & 20 \\
\hline
\end{tabular}


Table 2. Cont.

\begin{tabular}{|c|c|c|c|c|c|c|c|c|}
\hline Designated name & Unigene ID & $\begin{array}{l}\text { Nucleotide } \\
\text { length (nt) }\end{array}$ & $\begin{array}{l}\text { Full } \\
\text { length }^{a}\end{array}$ & $\begin{array}{l}\text { Protein } \\
\text { length }(\mathbf{a a})^{\mathrm{b}}\end{array}$ & $\begin{array}{l}\text { Predicted } \\
\text { signal peptide }^{c}\end{array}$ & $\begin{array}{l}\text { Fold } \\
\text { change }^{d}\end{array}$ & P value ${ }^{e}$ & $\begin{array}{l}\text { Identity to } \\
\text { best hit (\%) }\end{array}$ \\
\hline OfIKK $\beta$ & CL495.Contig1 & 1924 & $5^{\prime}$ & 613 & & 0.1559 & $2.82 \mathrm{E}-01$ & 34 \\
\hline \multicolumn{9}{|l|}{ Tak1 } \\
\hline OfTak1 & Unigene9186 & 875 & M & 291 & N/A & -1.0067 & $1.78 \mathrm{E}-04$ & 72 \\
\hline \multicolumn{9}{|l|}{ IAP2 } \\
\hline OfIAP2 & CL9344.Contig1 & 1872 & Yes & 566 & & 1.0766 & 2.09E-07 & 56 \\
\hline \multicolumn{9}{|l|}{ Tab2 } \\
\hline OfTab2 & CL6310.Contig2 & 2353 & Yes & 592 & & 0.5095 & 4.04E-03 & 65 \\
\hline \multicolumn{9}{|l|}{ (JAK/STAT pathway) } \\
\hline \multicolumn{9}{|l|}{ STAT } \\
\hline OfSTAT & CL7684.Contig1 & 2964 & $3^{\prime}$ & 721 & $\mathrm{~N} / \mathrm{A}$ & -0.419 & 1.44E-04 & 83 \\
\hline \multicolumn{9}{|l|}{ Domeless } \\
\hline OfDomeless & Unigene9848 & 3041 & $5^{\prime}$ & 919 & & 0.9573 & $4.25 \mathrm{E}-30$ & 29 \\
\hline \multicolumn{9}{|l|}{ PIAS } \\
\hline OfPIAS1 & CL862.Contig4 & 2465 & Yes & 653 & & 0.0151 & $9.76 \mathrm{E}-01$ & 76 \\
\hline OfPIAS2 & CL862.Contig5 & 2983 & Yes & 619 & & -0.6311 & $9.07 \mathrm{E}-03$ & 77 \\
\hline OfPIAS3 & CL862.Contig6 & 3033 & Yes & 614 & & 0.2136 & 7.71E-01 & 76 \\
\hline OfPIAS4 & CL862.Contig3 & 2181 & Yes & 580 & & -1.2089 & $3.99 \mathrm{E}-03$ & 77 \\
\hline \multicolumn{9}{|l|}{ SOCS } \\
\hline OfSOCS & Unigene9103 & 1543 & Yes & 354 & & -0.5117 & $3.38 \mathrm{E}-03$ & 81 \\
\hline \multicolumn{9}{|l|}{ Hopscotch } \\
\hline OfHopscotch & Unigene16260 & 1097 & $5^{\prime}$ & 323 & & -1.0724 & $4.60 \mathrm{E}-04$ & 38 \\
\hline \multicolumn{9}{|l|}{ Effectors } \\
\hline \multicolumn{9}{|c|}{ Prophenoloxidase (PPO) } \\
\hline OfPPO1 & CL5552.Contig1 & 1299 & $5^{\prime}$ & 407 & & -1.9558 & $2.20 \mathrm{E}-74$ & 77 \\
\hline OfPPO2 & CL997.Contig1 & 2366 & Yes & 692 & & -2.296 & $6.45 \mathrm{E}-207$ & 100 \\
\hline OfPPO3 & Unigene28348 & 1153 & $5^{\prime}$ & 365 & & -1.3114 & $5.38 \mathrm{E}-28$ & 70 \\
\hline \multicolumn{9}{|c|}{ (Antimicrobial peptide, AMP) } \\
\hline \multicolumn{9}{|l|}{ lysozyme } \\
\hline OfLys1 & Unigene9475 & 2426 & Yes & 155 & $1-25 G * Q$ & -0.5278 & $5.215 \mathrm{E}-12$ & 72 \\
\hline OfLys2 & CL5427.Contig1 & 905 & Yes & 140 & $1-20 A^{*} K$ & 2.6495 & 0 & 99 \\
\hline OfLys3 & Unigene20582 & 1732 & Yes & 140 & $1-20 A * V$ & 2.1514 & 0 & 87 \\
\hline OfLys4 & CL6947.Contig5 & 523 & $5^{\prime}$ & 137 & $1-16 G * V$ & -3.0725 & $1.59 \mathrm{E}-02$ & 69 \\
\hline OfLys5 & CL5801.Contig1 & 1027 & Yes & 143 & $1-20 G * R$ & 0.949 & $1.32 \mathrm{E}-16$ & 51 \\
\hline OfLys6 & Unigene13076 & 827 & Yes & 140 & $1-18 A^{*} F$ & -2.4845 & 0 & 66 \\
\hline OfLys-L1 & Unigene10659 & 780 & Yes & 186 & $1-17 G * R$ & 0.2146 & 1.07E-01 & 68 \\
\hline OfLys-L2 & CL9850.Contig2 & 1877 & Yes & 180 & $1-22 S^{*} \mathrm{~T}$ & -0.4783 & 4.10E-14 & 72 \\
\hline \multicolumn{9}{|l|}{ cecropin } \\
\hline OfCec1 & Unigene13269 & 547 & Yes & 68 & $1-22 A^{*} T$ & 2.7295 & 0 & 51 \\
\hline OfCec2 & CL1178.Contig1 & 2056 & Yes & 66 & $1-22 A^{*} A$ & 2.7421 & 0 & 73 \\
\hline OfCec3 & CL7041.Contig1 & 723 & Yes & 66 & $1-22 A^{*} A$ & 2.1301 & 0 & 66 \\
\hline OfCec4 & Unigene828 & 1332 & Yes & 65 & $1-22 A * S$ & 3.705 & 0 & 58 \\
\hline OfCec5 & Unigene14482 & 1186 & Yes & 64 & $1-22 A^{*} A$ & 3.0652 & 0 & 75 \\
\hline OfCec6 & Unigene22346 & 470 & Yes & 61 & $1-22 A^{*} A$ & 2.8013 & 0 & 78 \\
\hline \multicolumn{9}{|l|}{ defensin } \\
\hline OfDefensin & CL4424.Contig1 & 477 & Yes & 108 & $1-22 S^{*} \mathrm{~F}$ & 0.6963 & $9.80 \mathrm{E}-61$ & 33 \\
\hline \multicolumn{9}{|l|}{ attacin } \\
\hline OfAttacin & CL8975.Contig1 & 983 & Yes & 204 & $1-17 S^{*} Q$ & 1.4461 & 0 & 52 \\
\hline \multicolumn{9}{|l|}{ gloverin } \\
\hline OfGloverin1 & Unigene1854 & 1164 & Yes & 196 & $1-15 A^{*} Q$ & 1.5723 & 0 & 57 \\
\hline
\end{tabular}


Table 2. Cont.

\begin{tabular}{|c|c|c|c|c|c|c|c|c|}
\hline Designated name & Unigene ID & $\begin{array}{l}\text { Nucleotide } \\
\text { length (nt) }\end{array}$ & $\begin{array}{l}\text { Full } \\
\text { length }^{a}\end{array}$ & $\begin{array}{l}\text { Protein } \\
\text { length }(a a)^{b}\end{array}$ & $\begin{array}{l}\text { Predicted } \\
\text { signal peptide }^{c}\end{array}$ & $\begin{array}{l}\text { Fold } \\
\text { change }^{d}\end{array}$ & P value ${ }^{e}$ & $\begin{array}{l}\text { Identity to } \\
\text { best hit }(\%)^{f}\end{array}$ \\
\hline OfGloverin2 & CL516.Contig4 & 1384 & Yes & 198 & $1-15 A^{*} Q$ & 1.2401 & 0 & 57 \\
\hline \multicolumn{9}{|l|}{ Lebocin } \\
\hline OfLebocin 1 & CL8338.Contig1 & 1310 & Yes & 199 & $1-22 A^{*} Q$ & 2.4102 & 0 & 51 \\
\hline OfLebocin2 & CL8528.Contig2 & 391 & $5^{\prime}$ & 124 & $1-22 A^{*} Q$ & 1.7827 & 0 & 37 \\
\hline OfLebocin3 & CL8528.Contig1 & 386 & $5^{\prime}$ & 121 & $1-22 A^{*} Q$ & 1.285 & 0 & 37 \\
\hline \multicolumn{9}{|l|}{ Moricin } \\
\hline OfMoricin 1 & Unigene8386 & 388 & Yes & 63 & $1-23 A^{*} N$ & 4.0096 & 0 & 58 \\
\hline OfMoricin2 & Unigene4140 & 359 & Yes & 63 & $1-22 A^{*} N$ & 3.0459 & 0 & 53 \\
\hline OfMoricin3 & CL4055.Contig5 & 536 & Yes & 62 & $1-23 A^{*} N$ & 4.3116 & $9.00 \mathrm{E}-304$ & 66 \\
\hline OfMoricin4 & Unigene33399 & 239 & $5^{\prime}$ & 61 & $1-23 G * N$ & 3.4761 & $6.63 \mathrm{E}-12$ & 50 \\
\hline \multicolumn{9}{|c|}{ Nitric oxide synthase (NOS) } \\
\hline OfNOS1 & CL332.Contig1 & 2618 & $3^{\prime}$ & 777 & $\mathrm{~N} / \mathrm{A}$ & 1.1857 & $3.31 \mathrm{E}-16$ & 82 \\
\hline OfNOS2 & Unigene9115 & 1901 & $3^{\prime}$ & 575 & $\mathrm{~N} / \mathrm{A}$ & -0.1862 & $3.61 \mathrm{E}-01$ & 90 \\
\hline \multicolumn{9}{|l|}{ reeler } \\
\hline OfReeler & CL9361.Contig1 & 1064 & Yes & 165 & $1-18 G^{*} Y$ & 4.8393 & 0 & 42 \\
\hline \multicolumn{9}{|c|}{ NADPH oxidase (NOX) } \\
\hline OfNOX & Unigene18492 & 1617 & $M$ & 538 & $\mathrm{~N} / \mathrm{A}$ & -0.6201 & 0.0001625 & 59 \\
\hline \multicolumn{9}{|l|}{ Peroxidase (Pox) } \\
\hline OfPox1 & CL6829.Contig2 & 4356 & Yes & 1341 & $1-24 A^{*} Q$ & -0.3948 & $2.338 \mathrm{E}-06$ & 60 \\
\hline OfPox2 & Unigene13729 & 3539 & Yes & 798 & & -0.1627 & $1.416 \mathrm{E}-06$ & 74 \\
\hline OfPox3 & CL812.Contig1 & 2051 & Yes & 648 & $1-17 G * N$ & -2.1365 & $4.55 E-232$ & 54 \\
\hline OfPox4 & Unigene2378 & 2149 & Yes & 656 & $1-17 G * G$ & 2.4212 & $1.85 \mathrm{E}-123$ & 55 \\
\hline OfPox 5 & CL517.Contig7 & 2522 & Yes & 716 & $1-16 A^{*} L$ & -1.9023 & $7.623 \mathrm{E}-08$ & 53 \\
\hline OfPox6 & Unigene5009 & 1124 & $M$ & 374 & $\mathrm{~N} / \mathrm{A}$ & -2.5073 & $3.684 \mathrm{E}-11$ & 53 \\
\hline OfPox7 & Unigene26669 & 1049 & $3^{\prime}$ & 331 & $\mathrm{~N} / \mathrm{A}$ & -11.7799 & $9.637 \mathrm{E}-11$ & 69 \\
\hline OfPox8 & Unigene6370 & 933 & $3^{\prime}$ & 275 & $\mathrm{~N} / \mathrm{A}$ & -2.7268 & $2.448 \mathrm{E}-51$ & 48 \\
\hline OfPox9 & Unigene19867 & 818 & $M$ & 272 & $\mathrm{~N} / \mathrm{A}$ & 0.4346 & 0.165 & 46 \\
\hline OfPox10 & Unigene21448 & 821 & $3^{\prime}$ & 257 & $\mathrm{~N} / \mathrm{A}$ & 0.4738 & 0.089999 & 50 \\
\hline OfPox11 & Unigene935 & 1302 & Yes & 249 & $1-19 C^{*} \mathrm{~T}$ & -0.5707 & $4 \mathrm{E}-23$ & 92 \\
\hline OfPox12 & CL1677.Contig1 & 1085 & Yes & 227 & & 1.6154 & $1.459 \mathrm{E}-06$ & 82 \\
\hline OfPox13 & Unigene4211 & 991 & Yes & 223 & & -0.082 & 0.37496 & 83 \\
\hline OfPox14 & CL9355.Contig4 & 1490 & Yes & 198 & $1-18 T^{*} A$ & -0.7768 & 0.0084466 & 79 \\
\hline OfPox15 & CL9299.Contig1 & 1357 & Yes & 195 & & 0.3196 & $4.936 \mathrm{E}-11$ & 95 \\
\hline OfPox16 & CL3991.Contig2 & 684 & $3^{\prime}$ & 166 & $\mathrm{~N} / \mathrm{A}$ & 0.501 & 0.14413 & 43 \\
\hline OfPox17 & Unigene5118 & 571 & M & 190 & $\mathrm{~N} / \mathrm{A}$ & -0.9783 & 0.0004929 & 46 \\
\hline \multicolumn{9}{|c|}{ Superoxide dismutase (SOD) } \\
\hline OfSOD1 & Unigene16806 & 1130 & Yes & 286 & & 0.4061 & 0.0059053 & 65 \\
\hline OfSOD2 & Unigene22638 & 1010 & Yes & 241 & $1-18 A^{*} L$ & 0.5179 & 0.024544 & 77 \\
\hline OfSOD3 & Unigene4403 & 915 & Yes & 216 & & -0.6654 & $9.254 \mathrm{E}-54$ & 85 \\
\hline OfSOD4 & Unigene18267 & 621 & $5^{\prime}$ & 192 & $1-19 A^{*} D$ & -2.4673 & $3.312 \mathrm{E}-95$ & 55 \\
\hline OfSOD5 & CL5843.Contig2 & 1218 & Yes & 172 & $1-16 A^{*} H$ & -2.2343 & 0 & 64 \\
\hline OfSOD6 & CL9771.Contig2 & 1349 & Yes & 154 & & -0.0839 & 0.058992 & 89 \\
\hline \multicolumn{9}{|l|}{ Catalase } \\
\hline OfCAT & CL2411.Contig1 & 2051 & Yes & 550 & & -0.6635 & $1.57 \mathrm{E}-203$ & 93 \\
\hline \multicolumn{9}{|c|}{ Thioredoxin reductase } \\
\hline OfTrxR & CL3171.Contig1 & 1872 & Yes & 494 & & -0.1048 & 0.41271 & 71 \\
\hline \multicolumn{9}{|l|}{ Thioredoxin } \\
\hline OfTrx1 & Unigene21754 & 1356 & Yes & 286 & & -0.535 & $5.03 E-35$ & 86 \\
\hline OfTrx2 & CL5990.Contig3 & 953 & Yes & 153 & & -0.0055 & 0.98746 & 85 \\
\hline
\end{tabular}


Table 2. Cont.

\begin{tabular}{|c|c|c|c|c|c|c|c|c|}
\hline Designated name & Unigene ID & $\begin{array}{l}\text { Nucleotide } \\
\text { length (nt) }\end{array}$ & $\begin{array}{l}\text { Full } \\
\text { length }^{a}\end{array}$ & $\begin{array}{l}\text { Protein } \\
\text { length }(a a)^{b}\end{array}$ & $\begin{array}{l}\text { Predicted } \\
\text { signal peptide }^{c}\end{array}$ & $\begin{array}{l}\text { Fold } \\
\text { change }\end{array}$ & P value ${ }^{\mathbf{e}}$ & $\begin{array}{l}\text { Identity to } \\
\text { best hit (\%) }\end{array}$ \\
\hline OfTrx3 & CL6996.Contig1 & 1655 & Yes & 150 & & -0.5278 & $3.946 \mathrm{E}-11$ & 84 \\
\hline OfTrx4 & CL9041.Contig1 & 1238 & Yes & 106 & & 0.4083 & $2.998 \mathrm{E}-38$ & 97 \\
\hline \multicolumn{9}{|l|}{ Peroxiredoxin } \\
\hline OfPrx1 & Unigene935 & 1302 & Yes & 249 & $1-19 C^{*} \mathrm{~T}$ & -0.5707 & $4 \mathrm{E}-23$ & 92 \\
\hline OfPrx2 & CL1677.Contig1 & 1085 & Yes & 227 & & 1.6154 & $1.459 \mathrm{E}-06$ & 80 \\
\hline OfPrx3 & Unigene4211 & 991 & Yes & 223 & & -0.082 & 0.37496 & 84 \\
\hline OfPrx4 & CL9299.Contig1 & 1357 & Yes & 195 & & 0.3196 & 4.936E-11 & 92 \\
\hline OfPrx5 & CL8502.Contig1 & 1777 & Yes & 185 & & -1.4136 & $1.854 \mathrm{E}-73$ & 83 \\
\hline
\end{tabular}

${ }^{2}$ The mark of Yes, 5', 3', and $\mathrm{M}$ means that the fragment of the unigene consists of complete open reading frame, $5^{\prime}$-end containing start codon, $3^{\prime}$-end containing stop codon, and the middle part without start and stop codons, respectively.

${ }^{\mathrm{b}}$ The number indicates the length of amino acid sequences deduced from available coding region, which is complete or not.

'Blank, signal peptide unpredictable. N/A, truncated at amino-terminus.

${ }^{\mathrm{d}}$ The fold change is calculated by log2 (treated_RPKM/control_RPKM). The value no less than 1 or no more than -1 is defined as up-regulated or down-regulated, respectively.

e $\mathrm{P}$-value is determined by FDR (false discovery rate).

fIdentity indicates the percentage of identical amino acid residues in O. furnacalis unigenes to the sequence best hit in BLASTX.

doi:10.1371/journal.pone.0086436.t002

putative N-terminal $\beta$-1,3-glucan-recognition domain and the $\mathrm{C}$ terminal glucanase-like domain, but Of $\beta$ GRP4 lacked the $\mathrm{N}$ terminal $\beta$-1,3-glucan-recognition domain, suggesting that it is possibly unable to directly bind to $\beta$-1,3-glucan. Additionally, high sequences similarities were observed in the N-terminal domains of deduced $O$. furnacalis, Drosophila and Bombyx homologues. OfßGRP1-3 also comprise eight conserved $\beta$-strands (Figure 4A), suggesting their ability to bind to $\beta$-1,3-glucan. We performed the phylogenetic analysis for all Of $\beta$ GRP1- 4 and $42 \beta$ GRP sequences from other insect species to investigate the relationship between $O$. furnacalis $\beta$ GRPs and others. As shown in Figure $4 \mathrm{~B}$, all $46 \beta$ GRPs were clustered into two groups, one containing the active catalytic residues for $\beta$-1,3-glucanase activity and the other containing no such residues. Of $\beta$ GRP1-4 is presented as 1:1 orthologs to $B$. mori $\beta G R P 1-4$. The orthologs for OfßGRP1, OfBGRP2 and OfßGRP3 are present in lepidopterans but not in dipterans (Figure 4B). OfßGRP1 and OfBGRP3 are predicted to be paralogs and have expanded from a common ancestral gene after Lepidoptera had diverged (Figure 4B). The digital expression profiles showed $O f \beta G R P 2$ and OfBGRP4 mRNA level slightly increased while OfBGRP1 and OfBGRP3 were consistent in response to the infection of $B$. bassiana (Table 2). The qRT-PCR assay showed OfBGRP1 mRNA level decreased and OfBGRP4 expression kept unchanged after challenge (Figure 2).

C-type lectin (CTL) is probably the largest lectin family. They are a group of soluble and membrane-bound proteins that associate with carbohydrates in a $\mathrm{Ca}^{2+}$-dependent manner [64]. Invertebrate CTLs participate in immune responses including PPO activation [65], hemocyte-mediated nodule formation and encapsulation [66], opsonization and microbial clearance [67]. CTLs have a characteristic carbohydrate-binding domain (CRD) with a well-defined structure stabilized by two or three pairs of disulfide bonds [65]. Insect CTLs normally consist of tandem CRDs. In this study, we identified 14 CTL genes, and designated them as OfCTL1-14 (Table 2). All deduced O. furnacalis CTLs contain two consecutive CRDs, a characteristic for lepidopteran CTLs (Figure 5). Although $\mathrm{N}$ - and C-terminal CRDs from Hyphantria cunea lectin are reported to have different sugar-binding specificities, the detailed function of these CRDs is not yet clarified [68]. We postulated that the two CRDs of O. furnacalis CTLs also have different sugar-binding specificity and bind to different microorganisms. Except for CRDs, other structural modules have been identified in some CTLs. For example, Drosophila Furrowed (a Drosopohila CTL) and Bombyx CTL2 have Sushi motifs and transmembrane domain in addition to CRD $[31,69]$. We only found CRDs in OfCTL1-14 sequences. There might be some unknown CTLs we did not identify in the transcriptome. OfCTL6 has $56 \%$ amino acid sequence identity to $M$. sexta IML-2 which binds to lipopolysaccharide to stimulate hemocyte encapsulation and melanization $[64,65]$. This suggests that OfCTL6 might also play an important role as recognition receptor during the early phase of microbial infection. Phylogenetic analyses indicated that OfCTL1-9 only clustered with lepidopteran CTLs, and OfCTL10-14 grouped with both lepidopteran and dipteran CTLs (Figure 5). qRT-PCR assay revealed that the transcript level of OfCTL7 and OfCTL12 increased, OfCTL3 and OfCTL6 decreased, whereas OfCTL1 remained unchanged (Figure 2).

In addition, we also identified other PRP genes from $O$. furnacalis transcriptome, including 9 scavenger receptors (SCRs), 2 hemocytins, 1 hemolin, 2 galectins, 1 dscam, 1 Draper and 1 Eater (Table 2). The identification of a large number of PRP genes laid a good foundation for the further cloning and functional studies of PRPs in $O$. furnacalis.

Genes for extracellular signal modulation and amplification. After infectious parasites or pathogens are recognized by insects, the invasive signals are modulated by extracellular cascades. Similar to the coagulation pathway and complement system in human, insect plasma factors such as serine proteases $(\mathrm{SPs})$ and serine protease homologs $(\mathrm{SPHs})$ play critical roles in signal relaying/tuning and execution mechanisms $[31,70]$. SPs typically contains a catalytic triad consisting of His, Asp, and Ser residues, which are embedded in highly conserved sequence motif of TAAHC, DIAL and GDSGG, respectively [71]. SPHs lack proteolytic activity due to the substitution of the catalytic triad residues, but they can enhance specificities and catalytic activities of SPs [72-74]. SPs and SPHs constitute one of the largest protein families in insects $[27,75,76]$. We have identified 121 potential SP and SPH transcripts in $O$. furnacalis transcriptome. Given that the catalytic triad is vital to define a SP or SPH, we manually checked the deduced amino acid sequences of all 121 transcripts, and only 


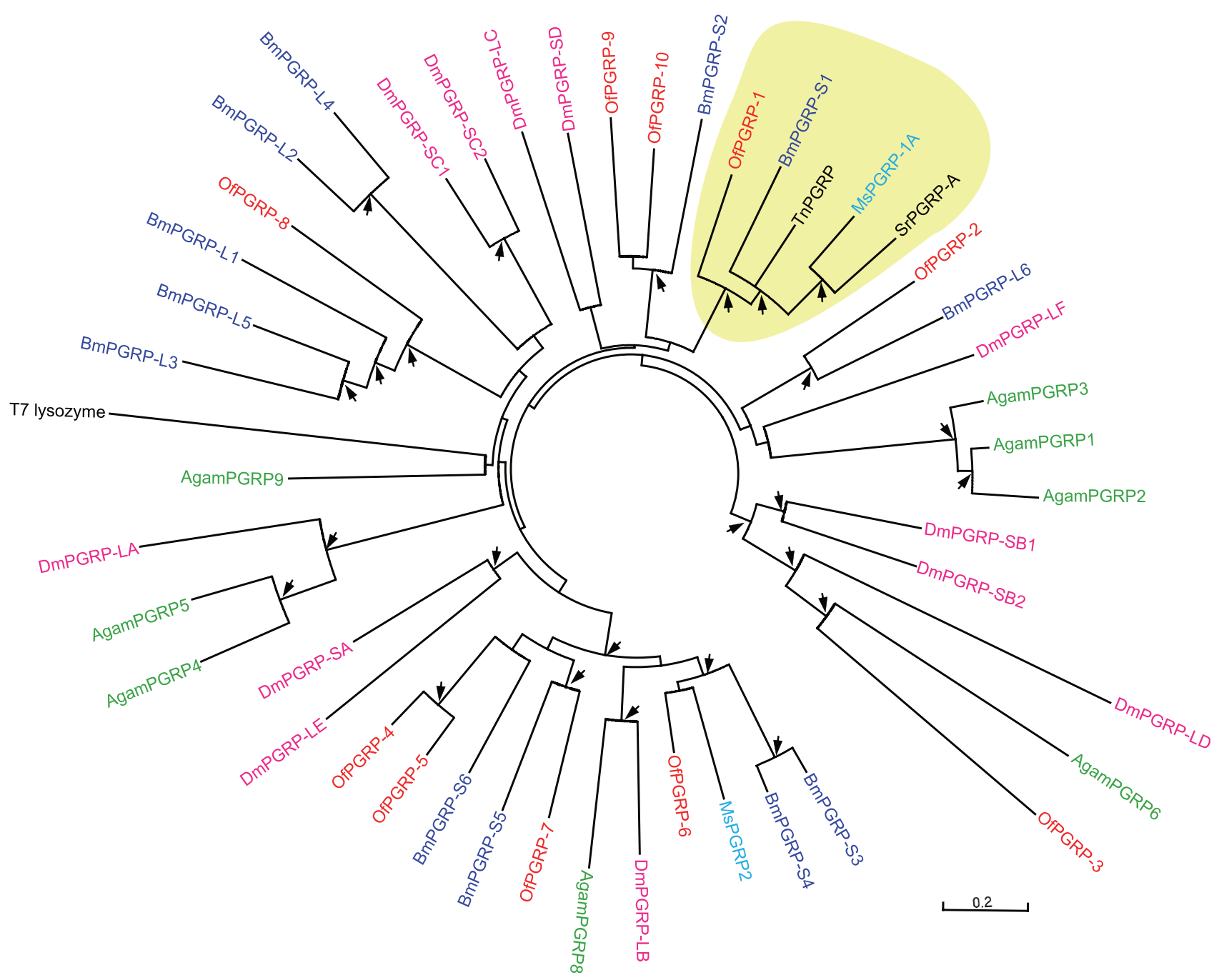

Figure 3. Phylogenetic analysis of peptidoglycan recognition proteins (PGRPs). The amino acid sequences from 10 Ostrinia (Of, red), 12 Drosophila (Dm, pink), 8 Anopheles (Ag, green), 12 Bombyx (Bm, purple), 2 Manduca (Ms, blue), one Samia ricini (Sr, black), one Trichoplusia ni (Tn, black) PGRPs, and T7 lysozyme (black) were used to build the unrooted tree. The clade that groups OfPGRP1 with other PGRPs known as recognition receptors in the PPO activation cascade (BmPGRP-S1 and MsPGRP-1A) was shaded in yellow. The arrows at nodes denote bootstrap value greater than 700 from 1000 trials.

doi:10.1371/journal.pone.0086436.g003

kept those containing all three sequence motifs. Finally we obtained $47 \mathrm{SP}$ (designated as OfSP1-OfSP47) and $14 \mathrm{SPH}$ (designated as OfSPH1-OfSPH14) transcripts. Fifty-six genes are predicted to have full length, 18 of which encode polypeptides with a SP or SP-like domain and other structural modules. These include 13 SPs (SP1-SP5, SP7, SP8, SP10, SP12-SP14, SP17 and SP37) and 3 SPHs (SPH8-SPH10) which contain one or more regulatory clip domains (Table 2), one SP (SP40) which contains a CUB domain, one SP (SP46) which contains five low density lipoprotein receptor A repeats (LDLa) domains and two complement control protein (CCP) domains. The clip domain is an important structural unit in which six conserved cysteine residues form three disulfide bonds [25,77]. In arthropods, clip-domain $\mathrm{SPs}$, and occasionally clip-domain $\mathrm{SPHs}$, are involved in many immune signaling pathways, such as melanization cascade and Toll pathway $[31,71,76]$. In $13 \mathrm{SPs}$ with clip domain, SP1 and SP13 mediated the immune responses of corn borer against $B$. bassiana by participating in the PPO activation cascade (submitted to Amino Acids). The other 11 clip-SPs are still under the investigation. Moreover, it is noteworthy that O. furnacalis SP46, with a large size and complex domain structure, is most similar to M. sexta HP14 [78] and T. molitor MSP [21] which both function as an initial enzyme to be recruited into the recognition complex in PPO activation cascade. We, therefore, inferred that SP46 also acts as the first enzyme in a serine protease pathway. Phylogenetic analysis indicated that $O$. furnacalis clip-domain proteins are divided into four subfamilies (Figure S5). The subfamily $\mathrm{A}$ is composed of SPHs solely while subfamilies B, C and D comprise SPs mainly. The four groups of SP-related genes may represent lineages derived from ancient evolutionary events since similar subfamilies also existed in Anopheles [27], Drosophila [75], and Tribolium [30].

Extracellular serine protease cascade are often regulated by members of the serine protease inhibitor (serpin) superfamily $[79,80]$. Serpins contain $\sim 400$ amino acid residues with an exposed reactive-center loop near their carboxyl terminus [81]. They inhibit serine proteases by forming covalent complexes, and therefore function as suicide-substrate inhibitors. Serpins have 
A

OfßGRP1 ---ORNQYKVPEAKIEATYPRGLVSIPDD-GFSLVAFHGKLNEEMEG-LEAGMWARDI OfßGRP2 VHNSRAQKFNI PDVKIEA FSPKGEAASI PDSPGMSLFVEQGNVNRAIDS -TAVGGIKGEI Of $\beta$ GRP3 -----RVDVP PAKLEAIYYKKLEVSIPDN-GESLFA FHGKLNEEMDG-LEAGEWISRDI BMßGRP1 ------YEAP PATLEA I HPKGLRVSVPDE-GFS LFA H HGKLNEEMEG-LEAGHWSRDI DMGNBP1 -------KIPTPTVELLET-GESVSIP DEE GVKVVA FNVNRNRNFTS F INEGOYNVR L

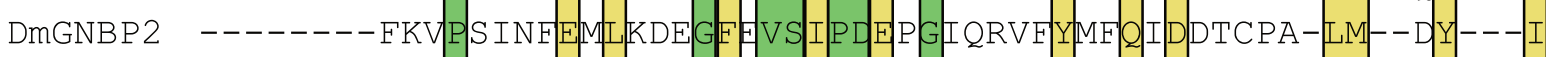

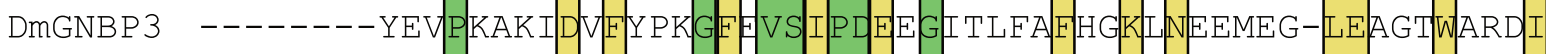
PißGRP --QPRAQQYVVPSAKLEAIYPKGLRVSIIPDD-GFSLFAEHGKLNEEMDG-LEAGHWARDI

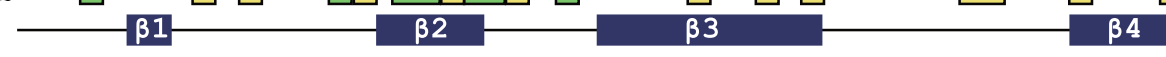

OfßGRP1 VRPKDGKFWRDRNIOUKLGDKVYFWTYAIKDGLGYRQDNGEWTVTEEVVNEK 106 Of 3 GRP2 TOATNGRNVYDNPDAQIKVGDVVNYYVEVISVNREGEVKDGLTYTITEFSSRS 111

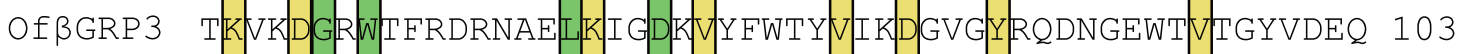
BmßGRP1 TKR PKNGRNITRDRNAA KKVGDKI Y FWTEVIKDGLGYRQDNGEWTVEGFVDEA 102

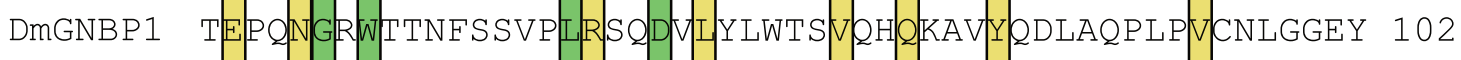
DmGNBP2 TEAVNGSWVSKQK-MS LENNDKL I SMLVVENEE I FEKSETRVI INTRLLTT 97 DMGNBP3 VKAAKNGRNIFRDRITAEKRGDTIYYWTYVI YNGLGYREDDGS FVVNGYSGNN 103 PißGRP TKPKEGRWTFRDRNVKLKLGDKIYFWTYUIKDGLGYRQDNGEWTUTEFVNED 108

B

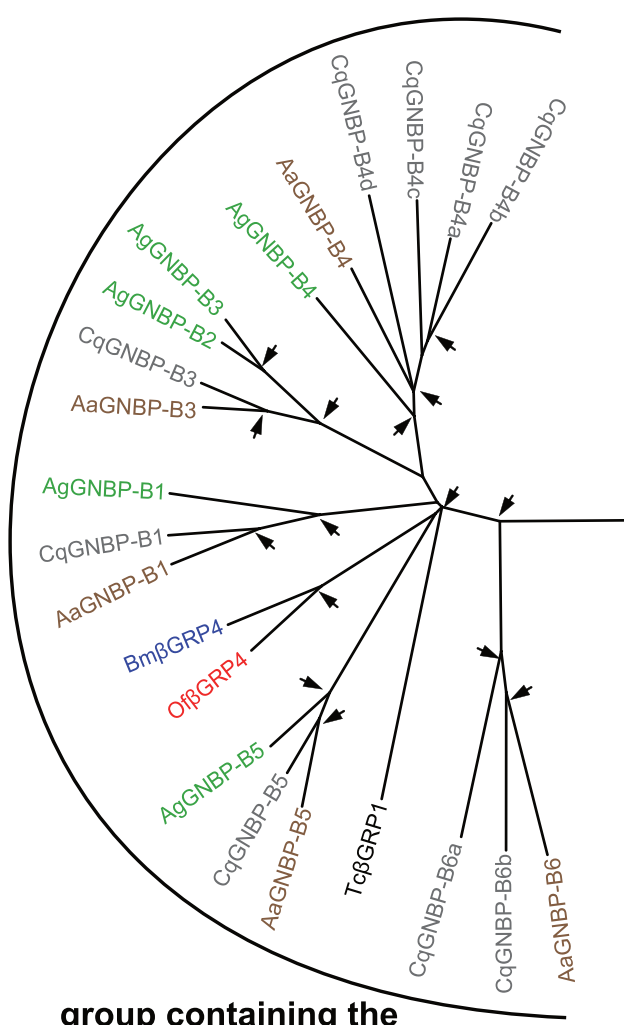

group containing the active site catalytic residues for $\beta$-1,3-glucanase activity

$\beta 6$

$\beta 7-\beta 8$

$B$ 
Figure 4. Alignments of the $\mathbf{N}$-terminal domains of $\boldsymbol{\beta}$-glucan recognition proteins ( $\boldsymbol{\beta G R P s )}$. (A) The deduced amino acid sequences of $O$. furnacalis $\beta$ GRP1-3 (Of $\beta$ GRP1-3) were compared with Drosophila GNBP1-3 (DmGNBP1-3), Bombyx $\beta$ GRP1 (Bm $\beta$ GRP1) and Plodia interpunctella $\beta$ GRP (PißGRP). The amino acids in green and yellow shade indicate the conserved and type-conserved residues, respectively. The predicted secondary structural elements of eight $\beta$-strands are shown below the alignments. (B) Phylogenetic relationships of $\beta$ GRPs. The aligned sequences of $\beta$ GRP family members were from Ostrinia (Of, red), Drosophila (Dm, pink), Anopheles (Ag, green), Aedes (Aa, brown), Culex quinquefasciatus (Cq, grey), Bombyx (Bm, purple), Manduca (Ms, blue), Helicoverpa armigera (Ha, orange), Trichoplusia (Tn, black), and Tribolium (Tc, black). Two divided groups are indicated by brackets. For explanation of the arrows see Fig. 6. doi:10.1371/journal.pone.0086436.g004

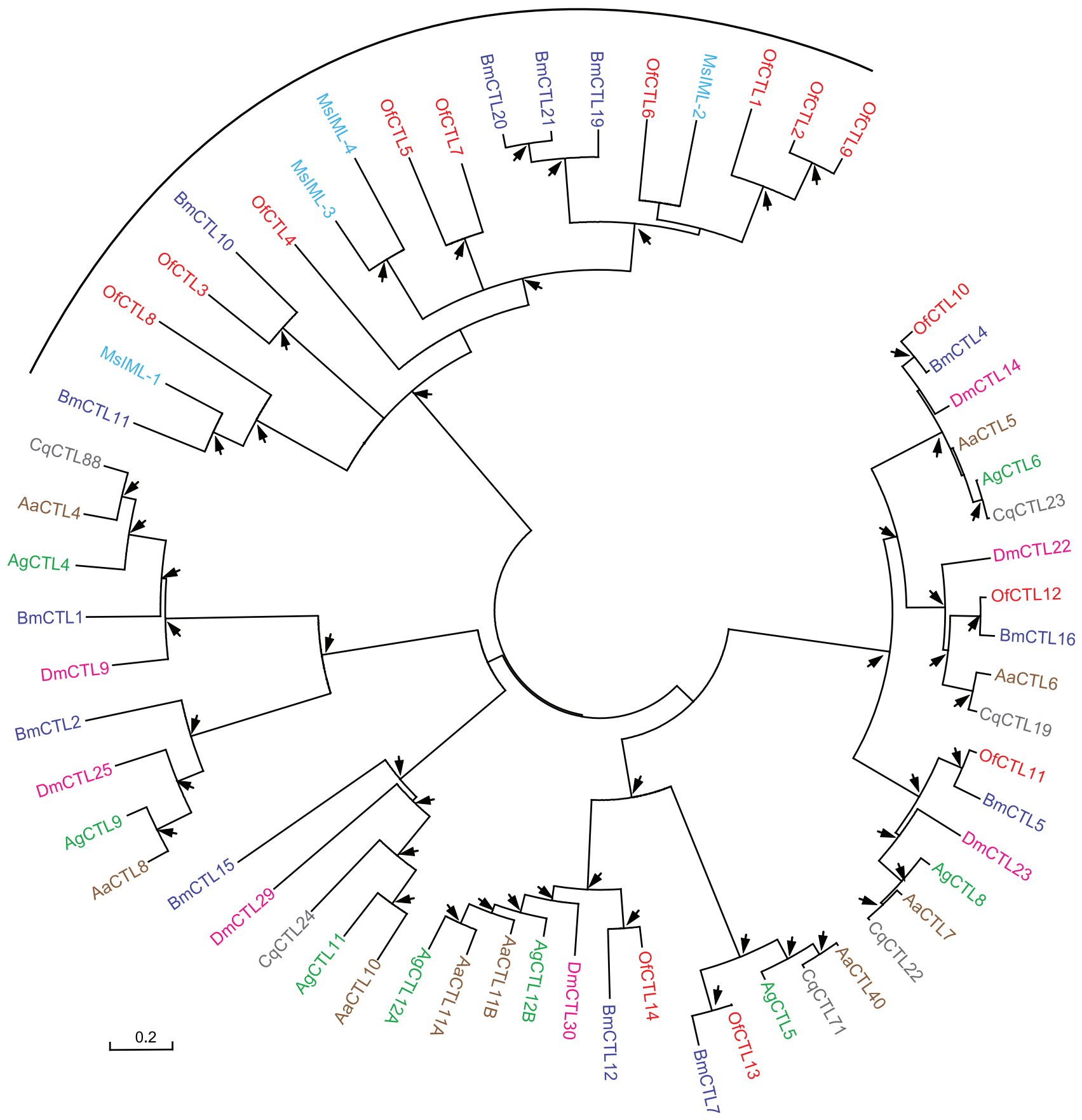

Figure 5. Phylogenetic analysis of C-type lectins (CTLs). The amino acid sequences of 61 CTLs from Ostrinia (Of, red), Drosophila (Dm, pink), Anopheles (Ag, green), Aedes (Aa, brown), Culex (Cq, grey), Bombyx (Bm, purple), and Manduca (Ms, blue) were examined. Lepidopteran-specific CTLs are indicted with bracket. For explanation of the arrows see Fig. 6.

doi:10.1371/journal.pone.0086436.g005 
been reported to participate in the regulation of melanization reactions in three different insect orders (flies, beetle, and moths), including Anopheles SRPN2 [82,83], Drosophila Spn27A [84,85], Manduca serpin-3 [86,87], and Tenebrio SPN40, SPN55 and SPN48 [88]. In this study, we have identified 17 serpin transcripts from $O$. furnacalis transcriptome, which were designated as OfSerpin 1A-1D, and 2 through 14 (Table 2). This includes four splicing variants for OfSerpin-1, in which only the last 40-53 amino acid residues in the reactive center loop are variable. Fourteen out of 17 serpin transcripts contain complete open reading frame with the exception of serpin-3, serpin-6, and serpin-14. Among 14 complete serpins, 13 ones consisted of a predicted signal peptide, suggesting they are secreted proteins. Serpin-2 lacks a secretion signal sequence, suggesting it might be an intracellular protein. In the phylogenetic analysis, OfSerpin 1-6s are presented as 1:1:1 orthologs to Manduca serpin 1-6 and Bombyx serpin 1-6, suggesting that $O$. furnacalis serpin1-6 has immune functions similar to Manduca and Bombyx serpins (Figure S6). It is noteworthy that one clade includes O. furnacalis serpin-3 as well as Anopheles SRPN2, Aedes Serpin-2, Drosophila Spn27A, Manduca serpin-3, and Bombyx serpin-3, which all functions as inhibitors of the melanization cascade [82,84-86]. Thus OfSerpin-3 may also regulate serine protease(s) involved in the melanization reaction. We analyzed the transcript change of OfSerpin-3 upon the challenge of B. bassiana conidia using qRT-PCR methods. The mRNA level of OfSerpin-3 significantly increased (Figure 2), also suggesting its putative role in regulating the immune response.

Genes involved in signal transduction. After the invasive signals from the infectious microorganisms are recognized and modulated, the signal transduction pathways will be initiated to produce the effector molecules. Four signal transduction pathways, Toll, Imd, JNK, and JAK/STAT are known to be involved in insect immunity [29]. Toll and Imd pathways are more important in sensing microbes. The Toll pathway is primarily involved in the defense against fungi and Gram-positive bacteria with lysine-type peptidoglycans (Lys-type PGNs) in their cell walls, while the Imd pathway responds to Gram-negative bacteria and some Grampositive bacteria with meso-di-aminopimelic acid-type peptidoglycans (DAP-type PGNs) [1]. In this study, we have identified at least 46 transcripts for signal transduction from $O$. furnacalis transcriptome, which nearly included all known components in signal transduction pathways (Table 2).

Spätzle is a ligand for the Toll receptor and activates the Toll signaling pathway [11]. Spätzle is present in Anopheles (6 genes) [27], Drosophila (6 genes) [26], and Bombyx (3 genes) [31]. Drosophila Spz1, Bombyx Spzl, and Manduca Spzl all have been demonstrated to participate in immunity [89-91]. ProSpätzle is consisted of an unstructured pro-domain and a C-terminal fragment that adopts a cysteine-knot structure similar to that of mammalian neurotrophins [92]. Six Spätzle genes have been identified from the transcriptome, with the tentative name as OfSpz1A, 1B, and 3-6. Only OfSpz1A, $1 B$, and -5 contains the complete open reading frame (Table 2). To assess the relationship between $O$. furnacalis Spätzle and other insect Spätzle proteins, we performed a phylogenetic analysis by aligning the known homologous cysteine-knot domain sequences from different insect species. The phylogenetic tree (Figure 6) suggests that all Spätzle homologs can be assigned to a 1:1 orthologous group with one of the Drosophila Spätzle gene products (Spz1-Spz6). No Spätzle-2 ortholog was identified in $O$. furnacalis transcriptome. A possible reason is that Spätzle-2 gene is missing in $O$. furnacalis because of the evolutionary event. The other reason with higher possibility is that the transcript level of Spätzle-2 is low and it is not captured in the RNA-seq. As shown in Figure 6, the bootstrap value at the clade including $\mathrm{Spz}-1$ is lower than in the other clades containing Spz-2 to Spz-6, indicating a lower degree of sequence conservation in Spätzle-1. It is worth noting that there are two possible $O$. furnacalis Spätzle-1 variants $(O f S p z-1 A$ and $O f S p z-1 B)$ in Spätzle-1 branch, which only share $33 \%$ identity in amino acid sequences. OfSpz-1A is more similar to other insect Spätzle-1, which had 56\% sequence similarities with Manduca Spätzle-1. qRT-PCR analysis indicated that the expression of both OfSpz-1A and OfSpz$1 B$ were induced by the $B$. bassiana conidia. It suggests that certain genes in Toll pathway are involved in innate immunity response against B. bassiana.

Toll receptor plays a crucial role in insect innate immune response and acts as the signal transducer in the Toll pathway [93]. It has been identified in many insect species, including the orthoptera, hymenoptera, coleoptera, lepidoptera and diptera. Toll-like receptors (TLR) have also been detected in mammals, suggesting that Toll and TLR are evolutionarily conserved throughout the insects and mammals [94]. Insect Toll receptors and mammalian TLRs are all type I membrane proteins with an ectodomain consisting of leucine-rich repeats (LRRs), a transmembrane domain, and an intracellular Toll-interleukin homolog domain (TIR) that can transduce signals [95]. In this study, we identified 12 genes coding Toll receptors in $O$. furnacalis transcriptome datasets. These genes were designated as OfToll112. Only OfToll1-4 and OfTollo genes contain the full-length encoding sequences (Table 2). Domain prediction with SMART revealed that among these 5 full-lengthed Toll-like genes, OfToll-1 through -3 is composed of the extracellular LRR, transmembrane and cytoplasmic TIR domains, while OfToll4 and OfToll8 only contains the extracellular LRRs and transmembrane domains (Figure 7). Among five incomplete $O$. furnacalis Toll-like genes with stop codon at $3^{\prime}$-end (OfToll5-7, -9, and -11), cytoplasmic TIR domain is only found in OfToll-5, OfToll-7, and OfToll-11 (Figure 7). We cannot conclude whether TIR domain is present in OfToll-10 and OfToll-12 because both 5'- and 3'-end of the cDNA sequences of these two genes are incomplete (Table 2 and Figure 7). On the other hand, the TIR domain has a more reliable determination of phylogeny than the extracellular LRR region [96]. Therefore, we were unable to conduct the convincible phylogenetic analysis for identified $O$. furnacalis Tolls. The efforts are being made to obtain the complete coding sequences for all potential $O$. furnacalis Toll genes. We investigated Toll gene expression after $B$. bassiana infection. Entopathogenetic fungus $B$. bassiana significantly increased the mRNA level of Toll-1, but decreased the transcript level of Toll-5 (Figure 2).

In Toll signaling pathway, the cysteine-knot part of Spätzle binds to the ectodomain of the Toll receptor and thereby triggers an intracellular signaling cascade including dMyD88, Tollip, Tube, Pelle, Pellino, TRAF2, and finally results in the degradation of inhibitor protein Cactus and the nuclear import of a rel family transcription factor Dorsal/Dif [9,97]. Contrary to the ligandreceptor diversification, components of the intracellular cascade appear to be highly conserved in insects [30]. In O. furnacalis transcriptome dataset, we have identified dMyD88, Tollip, Tube, Pelle, Pellino, TRAF2 with 1:1 orthologs (Table 2). We postulated that a similar protein complex also forms in O.furnacalis, which will lead to the release of a Rel transcription factor (CL7451.Contig1) from a cactus-like protein (CL1201.Contig1), allowing the Rel molecular to translocate into the nucleus and activate the expression of effector genes such as antimicrobial peptides (Figure S7).

Imd pathway is also composed of multiple molecules for the signal transduction. The involved intercellular components include Imd, Fas-associated death domain protein (FADD), Dredd, IAP2, 


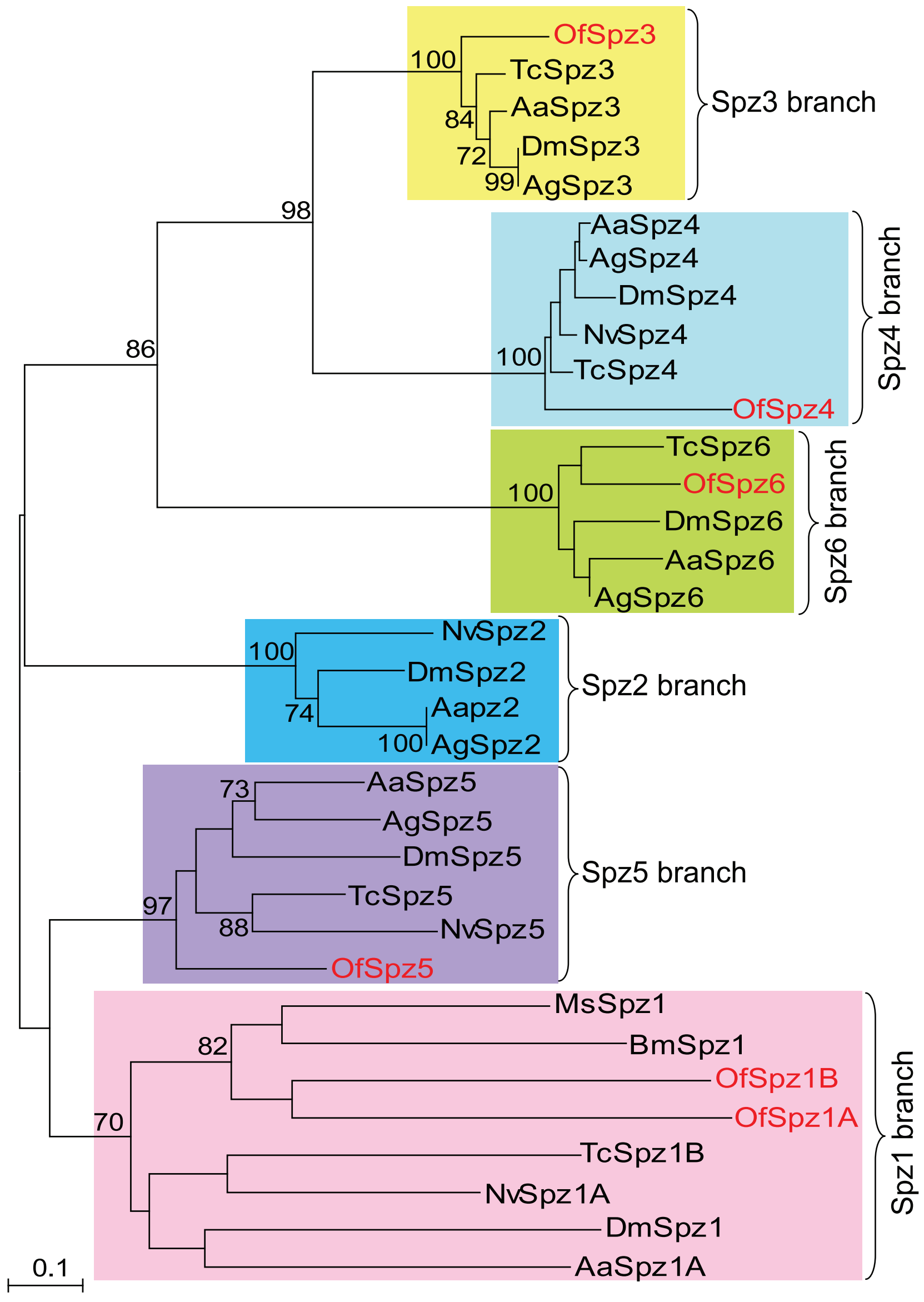


Figure 6. Phylogenetic analysis of the cysteine-knot domains in Spätzle from $O$. furnacalis and other insect species. The used amino acid sequences are from Ostrinia (Of, red), Drosophila (Dm), Anopheles (Ag), Aedes (Aa), Bombyx (Bm), Manduca (Ms), Tribolium (Tc), Nasonia vitripennis (Nv) cysteine-knot domains. Ostrinia Spzs are marked in red. The branches specific for Spz1 through Spz6 are shaded in squares. Numbers at the nodes are bootstrap values as percentage. Only bootstrap values greater than 70 are shown.

doi:10.1371/journal.pone.0086436.g006

transforming growth factor $\beta$ activated kinase (TAK1), Tab2, Ubc13, an inhibitor of nuclease factor $\kappa \mathrm{B}$ kinase subunits $\beta$ and $\gamma$ (IKK $\beta$ and $\operatorname{IKK} \gamma$ ), and a transcription factor Relish [98,99]. Orthologs of all these components were identified from $O$. furnacalis transcriptome (Table 2 and Figure S7). It strongly suggests that Imd-mediated immunity is conserved in Asian corn borer. Further experiments are required to verify the suggested role of each component in the immune response of $O$. furnacalis.

Genes for immune responsive effectors. Effector genes are induced in some specific tissues, such as fat bodies and hemocytes, following successive immune processes of signal recognition, modulation, and transduction. Part of the synthesized effector molecules are released into the hemolymph to play direct roles in phenoloxidase-dependent melanization, elimination of infectious microorganisms, apoptosis and other immune-related mechanism.

Prophenoloxidases (PPOs) are copper-containing enzymes. They are synthesized as inactive zymogens and activated by cleavage after residue arginine at approximately residue 50 [100,101]. A serine protease cascade contributes to the PPO activation. Active phenoloxidase (PO) catalyzed the hydroxylation of monophenols to 0 -diphenols and the oxidation of 0 -diphenols to quinones. Quinones are involved in microbial killing, melanin synthesis, sequestration of parasites or pathogens, and wound healing [7]. We have identified three PPO transcripts, and designated them as OfPPO1-3 (Table 2). The total number of PPO genes in different insect species did not change significantly, except for the mosquito Anopheles: 3, 9, 1, 2, 2, 2 PPO genes have been identified from Drosophila, Anopheles, Apis, Bombyx, Manduca, and Tribolium, respectively [31]. Among three identified PPO fragments, only OfPPO2 sequence was confirmed in other studies [102]. OfPPO2 is also the only one with complete coding region. Signal sequences of OfPPO1-3 are not confirmed, consistent with the situation in other insects. It suggests that PPOs are released from cells into the hemolymph by cell rupture [100]. In addition, PPO subunits form heterodimer in Bombyx (BomPPO1 and 2) and Manduca (MsPPO1 and 2), but form homodimer in Drosophila (DmDox-A3) [103]. We infer that Ostrinia PPO subunits can also form such heterodimer or homodimer. Phylogenetic analysis showed that $O$. furnacalis PPOs cluster well with other lepidopteran PPOs. The 1:1:1 orthologous relationship existed between OfPPO1, BmPPO1, and MsPPO1, as well as between OfPPO2, BmPPO2, and MsPPO2. It suggested that an ancestral PPO gene was duplicated after lepidoptera had diverged (Figure 8). qRT-

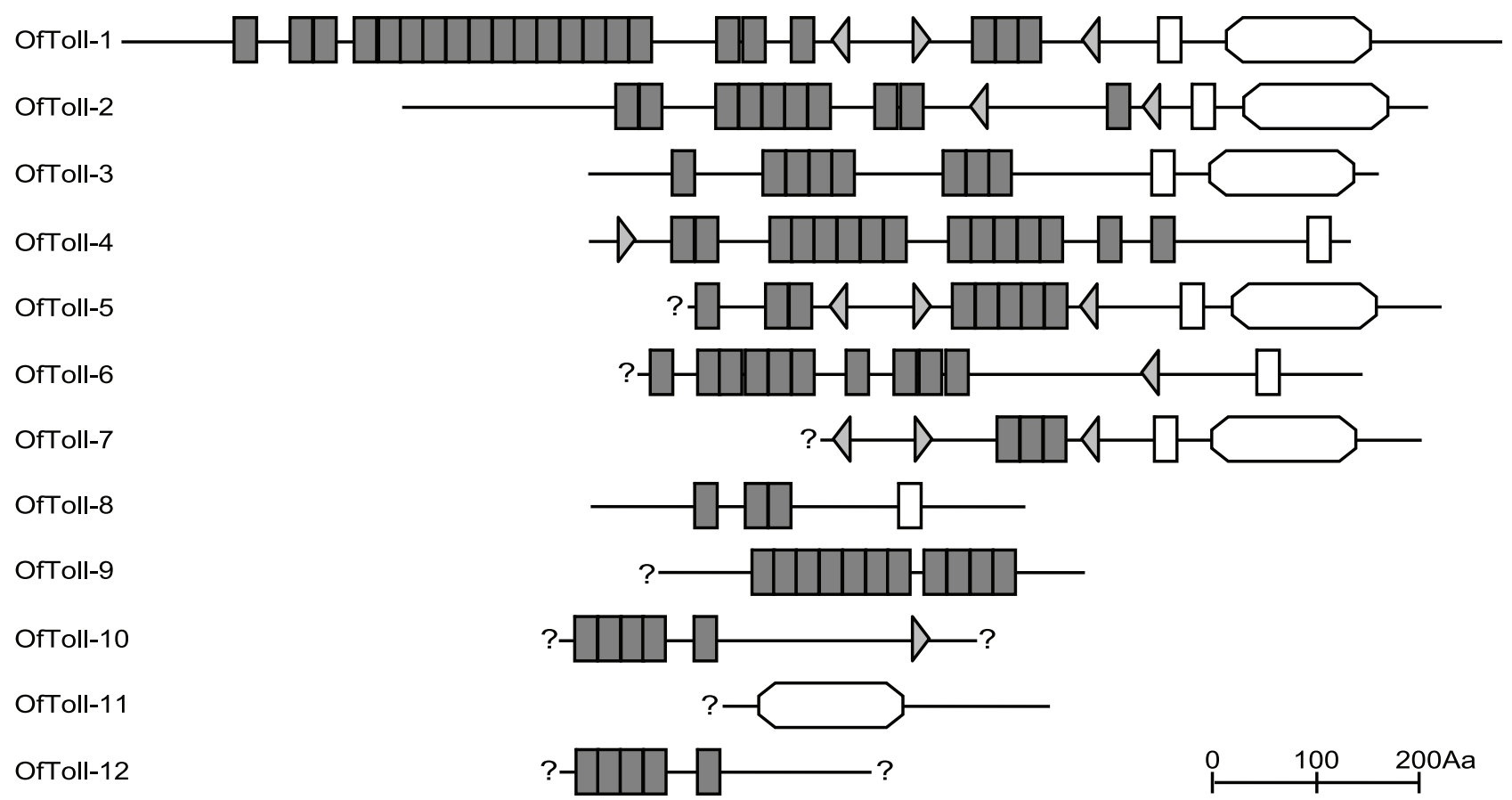

Leucine-rich repeat

Transmembrane domain $\checkmark$ Cysteine-rich carboxy-flanking motif

Cytoplasmic TIR domain
$D$ Cysteine-rich amino-flanking motif

? incomplete end

Figure 7. Schematic representation of the $\boldsymbol{O}$. furnacalis Tolls. The domain organization was predicted using the SMART program (http://smart. embl.de/). The extracellular leucine-rich repeats are shown by small rectangles. Cysteine-rich carboxy-flanking, and amino flanking motifs are shown by left and right triangles, respectively. Putative transmembrane domains are shown by open boxes. TIR domains are represented as hexagons. Question mark means the end is incomplete.

doi:10.1371/journal.pone.0086436.g007 


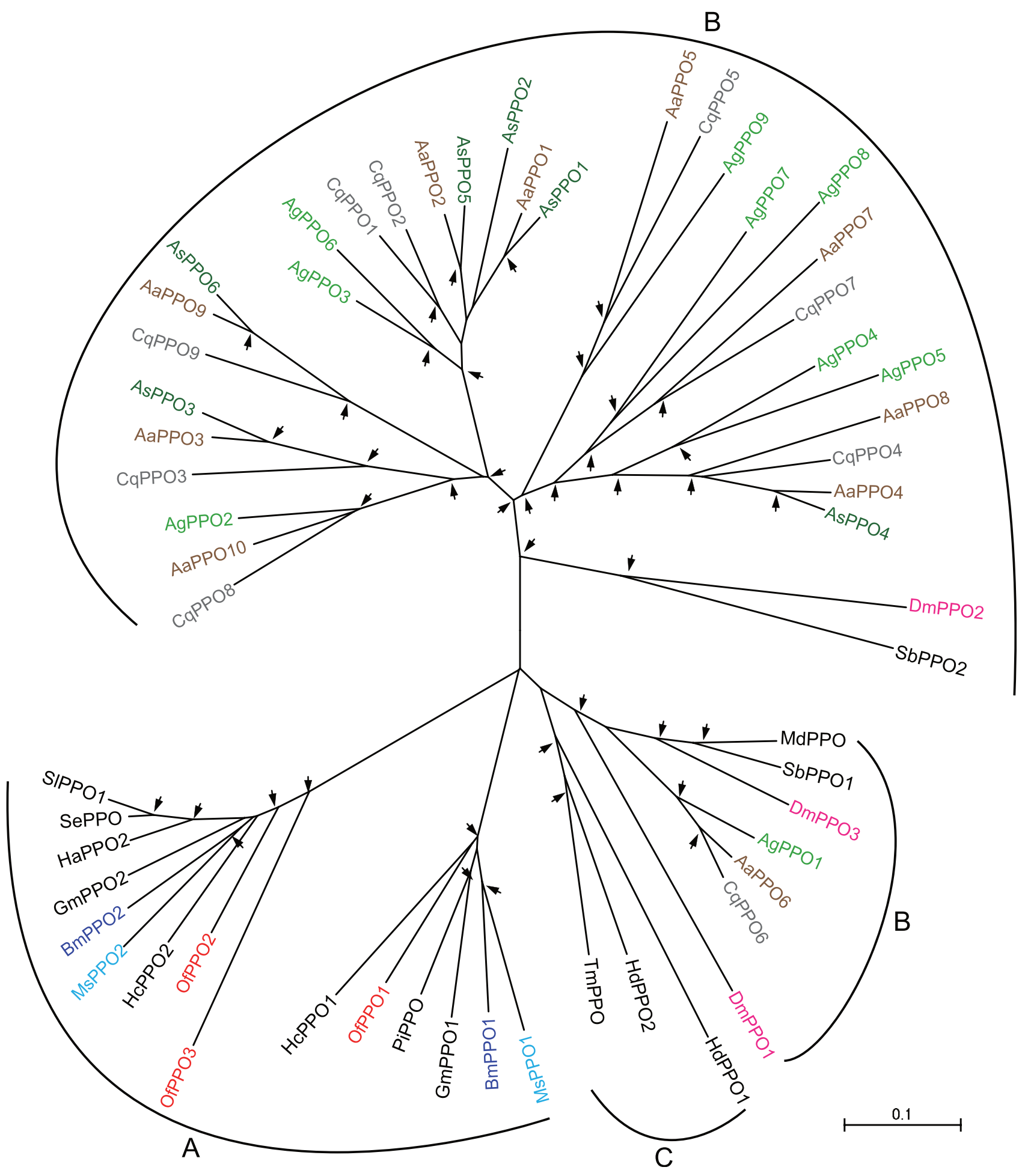

Figure 8. Phylogenetic analysis of prophenoloxidases (PPOs). The used amino acid sequences of 58 PPOs are from Ostrinia (Of), Drosophila $(\mathrm{Dm})$, Anopheles (Ag), Aedes (Aa), Culex (Cq), Bombyx (Bm), Manduca (Ms), Sarcophaga bullata (Sb), Musca domestica (Md), Armigeres subalbatus (As), Galleria mellonella $(\mathrm{Gm})$, Helicoverpa armigera $(\mathrm{Ha})$, Hyphantria cunea $(\mathrm{Hc})$, Holotrichia diomphalia $(\mathrm{Hd})$, Plodia interpunctella $(\mathrm{Pi})$, Spodoptera exigua (Se), Spodoptera litura (SI), Tenebrio molitor (Tm). The 1:1 and 1:1:1 orthologs including OfPPOs are present in the tree. A-C denotes the cluster specific for lepidopteran, dipteran, and coleopteran PPOs, respectively. For explanation of the arrows see Fig. 6. doi:10.1371/journal.pone.0086436.g008

PCR analysis of $O f P P O 2$ expression revealed that the mRNA level of $O f P P O 2$ was significantly increased after the injection of $B$. bassiana conidia (Figure 2).
Antimicrobial peptides (AMPs) are another group of immuneresponsive effectors that are directly active against the infectious microorganisms. Based on their amino acid composition and antimicrobial activities, AMPs are generally classified into five 


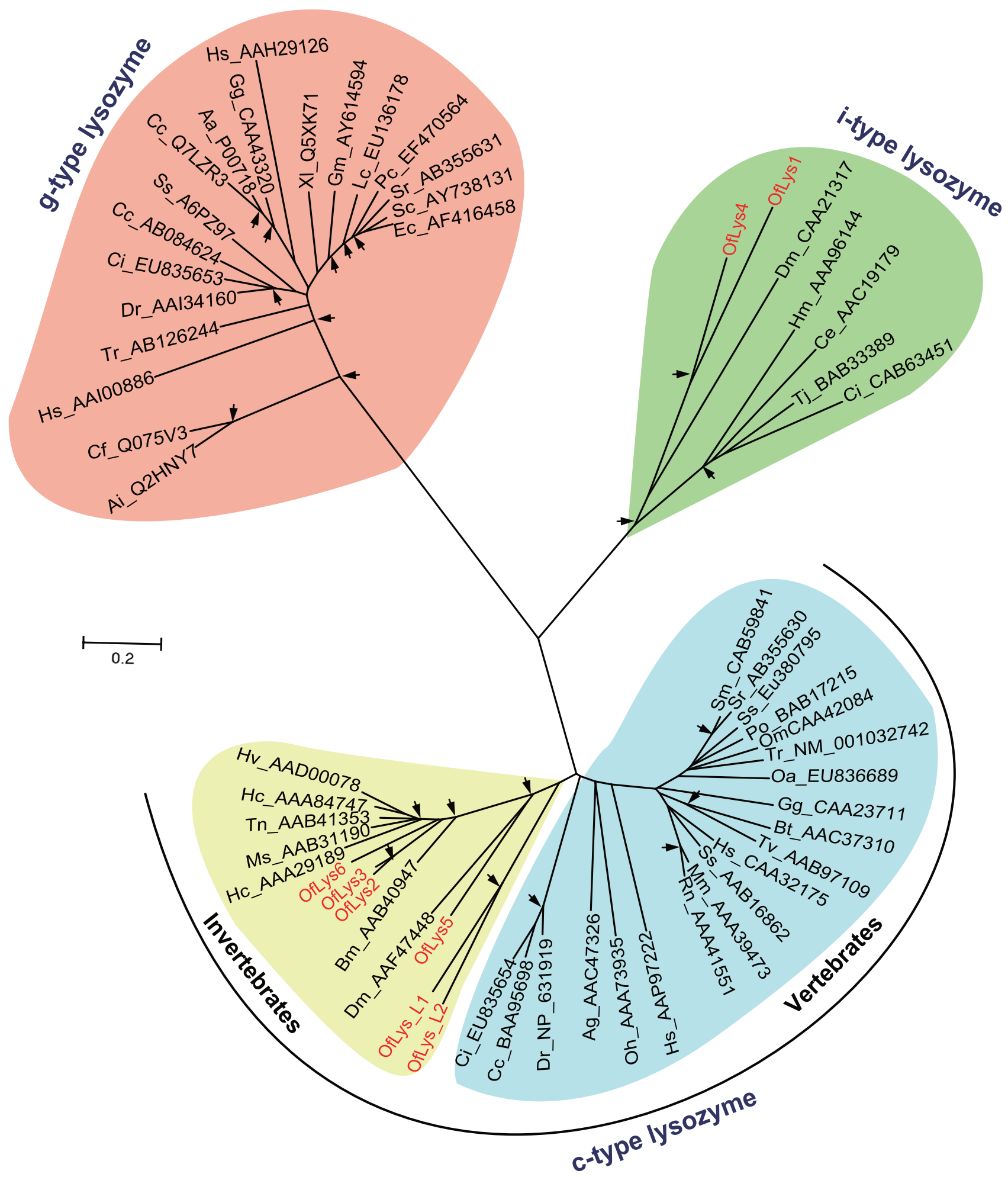

Figure 9. Phylogenetic analysis of lysozymes. The names of lysozyme genes used in the analysis were shown as scientific name of species followed by GenBank accession number of this specific gene. The Ostrinia lysozymes are marked in red. The branches specific for invertebrate c-type, vertebrate c-type, i-type, and g-type lysozymes are shaded in yellow, blue, green, and orange, respectively. For explanation of the arrows see Fig. 6. doi:10.1371/journal.pone.0086436.g009

groups: cecropins, insect defensins, lysozymes, proline-rich proteins, and glycine-rich proteins such as attacin [104,105]. Analysis of the $O$. furnacalis transcriptome revealed a large number of unigenes with homology to the major families of AMPs such as lysozymes, cecropins, moricins, lebocins, gloverins, defensins, and attacins (Table 2).

Lysozymes catalyze the hydrolysis of the $\beta$-1,4-glycosidic bond between $\mathrm{N}$-acetylglucosamine and $\mathrm{N}$-acetylmuramic acids of 
A

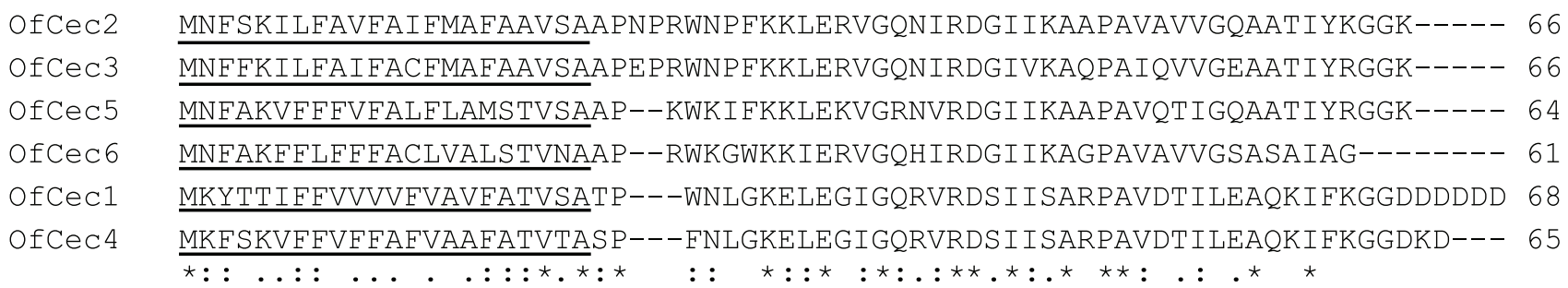

B

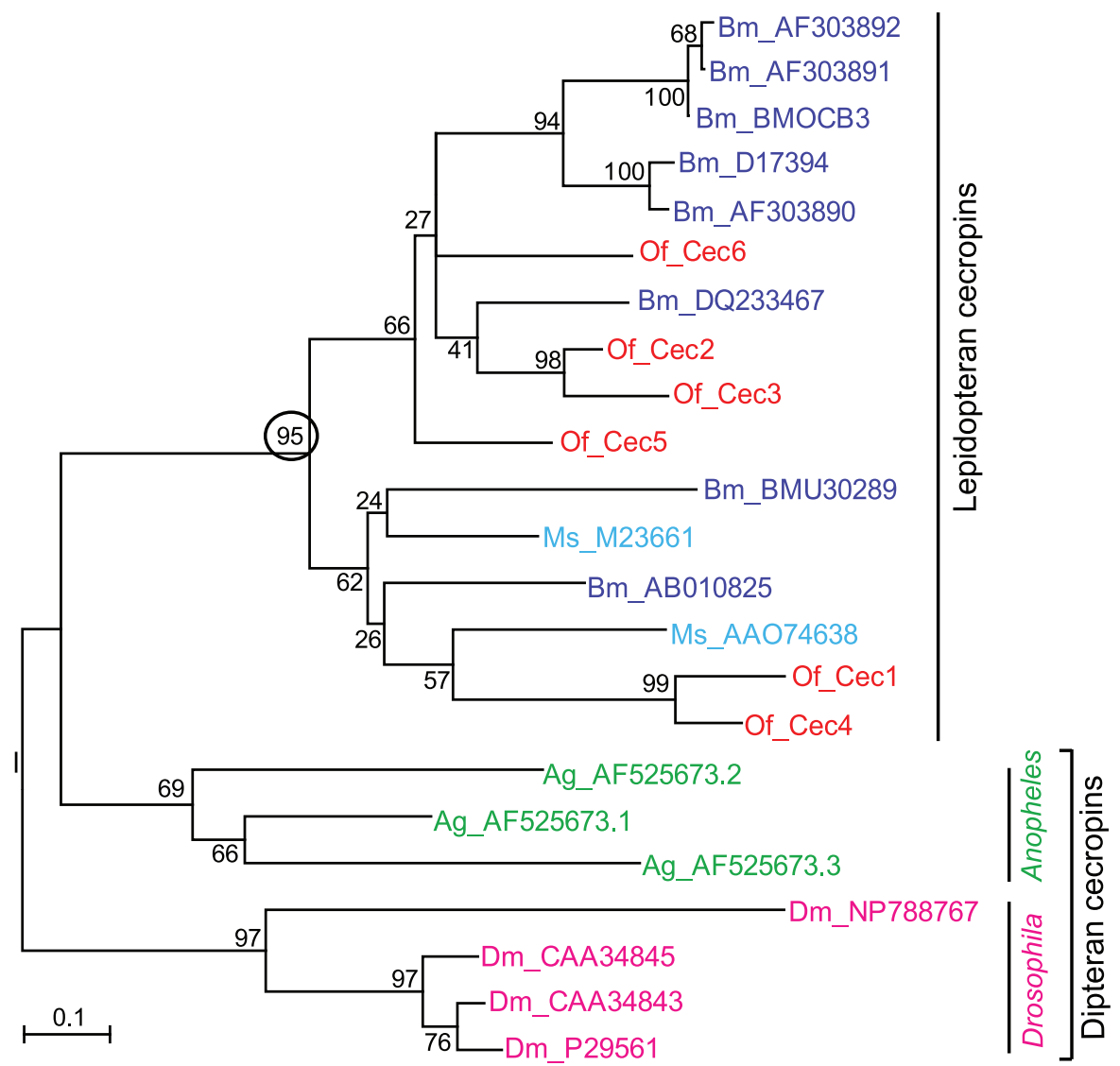

Figure 10. Alignment of full-length $\boldsymbol{O}$. furncalis cecropins. (A) Completely conserved amino acids are indicated by ' ${ }^{*}$, conservative substitutions by ' $:$ ', and semiconserved substitutions by '? below the sequences. The predicted secretion signal peptide is underlined. (B) Phylogenetic analysis of cecropins. The amino acid sequences from 8 Ostrinia (Of, red), 4 Drosophila (Dm, pink), 3 Anopheles (Ag, green), 8 Bombyx (Bm, purple), and 2 Manduca (Ms, blue) cecropins were used to build the neighbor-joining tree. Numbers at the nodes are bootstrap values as percentage. Only bootstrap values greater than 70 are shown. The circled bootstrap value indicates that Ostrinia cecropins belong to lepidopteran cecropins.

doi:10.1371/journal.pone.0086436.g010

peptidoglycans in bacterial cell walls [106]. They are divided into five major types including the c-type (chicken type), g-type (goose type), i-type (invertebrates), phage type, bacteria type, and plant type [107]. In this study, we identified six putative lysozyme genes (OfLys 1-Lys6), and two putative lysozyme-like genes (OfLys-L1 and OfLys-L2). OfLys-L1 and OfurLys-L2 have an amino acid replaced at one or both of catalytic amino acid position (glutamic acid and aspartic acid), and, therefore, may lose muramidase activity [108]. The phylogenetic analysis revealed that the c-, g- and i-type lysozymes form three independent clusters, respectively (Figure 9). Among c-type cluster, the invertebrate and vertebrate formed a subgroup respectively. OfLys1-OfLys4 is included in invertebrate c-type lysozyme subgroup. OfLys5 and OfLys6 are clustered with i-type lysozymes with a high bootstrap support. We randomly selected OfLys 2 for qRT-PCR assay. The result indicated that the expression of OfLys 2 was significantly up-regulated upon the challenge of B. Bassiana (Figure 2).

Cecropins represent another group of linear and amphipathic peptides which adopt a-helical structure. The first cecropin was discovered in Hyalophora cecropia [109]. They are widely present and active against Gram-negative and Gram-positive bacteria and fungi [110]. Both Drosophila and Anopheles contain 4 cecropin genes and Apis has none [27,29]. However, up to 13 cecropin genes was detected in Bombyx genome [31]. In this study, we have identified six putative cecropin genes, designated as OfCec1-OfCec6 (Table 2). All identified Ostrinia cecropin genes contain complete coding 
regions, and their deduced amino acid sequences have a predicted signal peptide with 22 residues. Alignment of the conceptive protein sequences of OfCecl-6 showed that they have high similarities. For example, OfCecl and OfCec4 share $82 \%$ identical amino acid residues, OfCec2 and OfCec3 have $85 \%$ identity in amino acid sequences (Figure 10A). Similar to the situation observed in the cecropin family in Spodoptera exigua [34], it is difficult to clearly distinguish that the sequence variations represent either different genes or different alleles (or alternative transcripts) from the same gene. Phylogenetic analysis showed that Drosophila, Anopheles and lepidopteran insects form cecropin gene clusters (Figure 10B). qRT-PCR assay for OfCec3 showed that the expression level of this gene significantly increased in response to B. bassiana infection (Figure 2). Other than lysozyme and cecropin genes, we have identified several other unigenes encoding putative AMPs in O. furnacalis transcriptome dataset, including four moricins, three lebocins, two gloverins, one defensin and one attacin (Table 2). Expression profile analysis with qRT-PCR methods showed that all tested antimicrobial peptide genes were significantly induced in response to the $B$. bassiana injection, with the exception of defensin remaining unchanged (Figure 2).

In addition, oxygen-derived free radicals and gaseous radical nitric oxide are also potent immune effector molecules [111]. Therefore, genes encoding the enzymes for the conversion of reactive oxygen species (ROS) and reactive nitrogen species (RNS) are somewhat classified as immune responsive effector genes. These enzymes include peroxidase (Pox), glutathione oxidase (GTX), superoxide dismutase (SOD), catalase, thioredoxin, thioredoxin reductase, peroxiredoxin, nitric oxide synthase (NOS), NADPH oxidase (NOX) and so on [30]. We have identified some of these genes in $O$. furnacalis transcriptome, including 17 Pox, 6 SOD, 1 catalase, 4 thioredoxin, 1 thioredoxin reductase, 5 peroxiredoxin, 2 NOS, and 1 NOX (Table 2). This suggests that local production of free radicals might be also a critical component of the immune response in Asian corn borers.

\section{Conclusions}

In summary, we sequenced and characterized the transcriptome from water-injected and B. bassiana-injected Asian corn borers. The transcriptome datasets obtained in this study make a significant contribution to a comprehensive sequence source for future $O$. furnacalis study, especially under the situation where its genomic information is currently unavailable. The explored immunity-related genes constitute an integrated picture of the immune network, which provides the valuable clues for a better understanding of the immune processes in $O$. furnacalis against $B$. bassiana. Immune constituent genes involved in signal recognition, modulation, transduction, and effector mechanisms have been identified and analyzed from the transcriptome. These immune repertoire genes appear to be evolutionarily conserved to different extent, and have various transcriptal profiles in response to the infection of B. bassiana. Functional analyses are necessary to verify our predictions. Nevertheless, the framework of information presented in this study should help clarify immune functions in an important agriculture pest and further understand the complex interaction between the insect pest and its entomopathogenic fungus.

\section{Supporting Information}

Figure S1 Homology analysis of $O$. furnacalis unigenes. (A) E-value distribution. (B) Similarity distribution. (C) Species distribution. All unigenes that had BLASTX annotations within the NCBI Nr database with a cut-off E-value of 10-5 were analyzed. The first hit of each sequence was used for analysis.

(EPS)

Figure S2 Gene ontology (GO) assignment for the $O$. furnacalis transcriptome. GO assignments (level 2) as predicted for their involvement in (A) biological processes, (B) cellular components, and $(\mathrm{C})$ molecular functions. The number of unigenes assigned to each GO term is shown behind semicolon. (EPS)

Figure S3 Clusters of orthologous groups (COG) classification of $\boldsymbol{O}$. furnacalis unigenes. A total of 11,462 produced functional annotations were among the 25 categories. The $\mathrm{Y}$-axis shows the number of unigene in each COG term.

(EPS)

Figure S4 Deduced amino acid sequences of 190 putative immunity-related unigenes. The coding region sequences (CDS) were determined on the base of the BLAST results. The amino acid sequences of each unigene were deduced in the EXPASY proteomics server (http://www.expasy.org). All sequences were listed in fasta format.

(TXT)

Figure S5 Phylogenetic analysis of catalytic domains from clip domain serine proteases (clip-SPs) and serine protease homologues (clip-SPHs). The amino acid sequences of 16 Ostrinia (Of, red), 40 Drosophila (Dm, pink), 45 Anopheles (Ag, green), 13 Bombyx (Bm, purple), 16 Manduca (Ms, blue), two Tenebrio molitor (Tm, black), three Holotrichia diomphalia (Hd, black), and two Limulus polyphemus (Lp, black) clip-SPs and clip-SPHs were used to build the unrooted tree. A-D denotes subfamilies of insect clipSPs. The arrows at nodes denote bootstrap value greater than 700 from 1000 trials.

(EPS)

Figure S6 Phylogenetic relationships among serpins (Spns). The amino acid sequences of 17 Ostrinia (Of, red), 6 Drosophila (Dm, pink), 5 Anopheles (Ag, green), 5 Aedes (Aa, brown), 28 Bombyx (Bm, purple), 6 Manduca (Ms, blue), 6 Tribolium (Tc, light purple); 4 Apis mellifera (Am, black) serpins were analyzed. The clade that groups OfSerpin-3 with other known melanization inhibitors including AgSRPN2, AaSpn2, DmSpn27A, and MsSerpin-3 was shaded in yellow. The arrows at nodes denote bootstrap value greater than 700 from 1000 trials.

(EPS)

Figure S7 Schematic drawing of the Toll (A) and Imd (B) signaling pathway in Drosophila and Ostrinia. Components of the putative pathway from $O$. furnacalis are predicted based on sequence similarity. The Drosophila gene names are followed by unigene number of their Asian corn borer orthologs (or paralogs in some cases).

(EPS)

Table S1 Primers for qRT-PGR analysis. (XLS)

Table S2 Statistics of sequencing quality. (DOC)

Table S3 The ID, length, expression and functional annotation of each unigene. (XLSX)

Table S4 The statistics of annotated unigenes. (DOG) 
Table S5 The ID, length, expression and functional annotation of each differently expressed unigene. (XLS)

\section{References}

1. Cherry S, Silverman N (2006) Host-pathogen interactions in drosophila: new tricks from an old friend. Nat Immunol 7: 911-917.

2. Jiang H, Vilcinskas A, Kanost MR (2010) Immunity in lepidopteran insects. Adv Exp Med Biol 708: 181-204.

3. Kanost MR, Nardi JB (2009) Innate immune response of Manduca sexta. In: Goldsmith MR, Marec F (eds) CRC Press, New York, PP. 271-291.

4. Aggarwal K, Silverman N (2008) Positive and negative regulation of the Drosophila immune response. BMB Rep 41: 267-277.

5. Iwanaga S, Lee BL (2005) Recent advances in the innate immunity of invertebrate animals. J Biochem Mol Biol 38: 128-150.

6. Lemaitre B, Hoffmann J (2007) The host defense of Drosophila melanogaster. Annu Rev Immunol 25: 697-743.

7. Cerenius L, Lee BL, Soderhall K (2008) The proPO-system: pros and cons for its role in invertebrate immunity. Trends Immunol 29: 263-271.

8. Kanost MR, Gorman MJ (2008) Phenoloxidases in insect immunity. Insect Immunology: 69-96.

9. De Gregorio E, Spellman PT, Tzou P, Rubin GM, Lemaitre B (2002) The Toll and Imd pathways are the major regulators of the immune response in Drosophila. The EMBO journal 21: 2568-2579.

10. Kambris Z, Brun S, Jang IH, Nam HJ, Romeo Y, et al. (2006) Drosophila immunity: a large-scale in vivo RNAi screen identifies five serine proteases required for Toll activation. CB 16: 808-813.

11. Ferrandon D, Imler JL, Hetru C, Hoffmann JA (2007) The Drosophila systemic immune response: sensing and signalling during bacterial and fungal infections. Nat Rev Immunol 7: 862-874.

12. El Chamy L, Leclerc V, Caldelari I, Reichhart JM (2008) Sensing of 'danger signals' and pathogen-associated molecular patterns defines binary signaling pathways 'upstream' of Toll. Nat Immunol 9: 1165-1170.

13. Tang H, Kambris Z, Lemaitre B, Hashimoto C (2006) Two proteases defining a melanization cascade in the immune system of Drosophila. J Biol Chem 281: 28097-28104.

14. Nam HJ, Jang IH, You H, Lee KA, Lee WJ (2012) Genetic evidence of a redox-dependent systemic wound response via Hayan protease-phenoloxidase system in Drosophila. EMBO J 31: 1253-1265.

15. Clark KD, Strand MR (2013) Hemolymph Melanization in the Silkmoth Bombyx mori Involves Formation of a High Molecular Mass Complex That Metabolizes Tyrosine. J Biol Chem 288: 14476-14487.

16. Shao Q Yang B, Xu Q Li X, Lu Z, et al. (2012) Hindgut innate immunity and regulation of fecal microbiota through melanization in insects. J Biol Chem 287: 14270-14279.

17. An C, Ishibashi J, Ragan EJ, Jiang H, Kanost MR (2009) Functions of Manduca sexta hemolymph proteinases HP6 and HP8 in two innate immune pathways. J Biol Chem 284: 19716-19726.

18. Gorman MJ, Wang Y, Jiang H, Kanost MR (2007) Manduca sexta hemolymph proteinase 21 activates prophenoloxidase-activating proteinase 3 in an insect innate immune response proteinase cascade. J Biol Chem 282: 11742-11749.

19. Jiang H,Wang Y, Yu XQ, Kanost MR (2003) Prophenoloxidase-activating proteinase-2 from hemolymph of Manduca sexta, A bacteria-inducible serine proteinase containing two clip domains. J Biol Chem 278: 3552-3561.

20. Lee SY, Kwon TH, Hyun JH, Choi JS, Kawabata SI, et al. (1998) In vitro activation of pro-phenol-oxidase by two kinds of pro-phenol-oxidase-activating factors isolated from hemolymph of coleopteran, Holotrichia diomphalia larvae. FEBS 254: 50-57.

21. Kim CH, Kim SJ, Kan H, Kwon HM, Roh KB, et al. (2008) A three-step proteolytic cascade mediates the activation of the peptidoglycan-induced toll pathway in an insect. J Biol Chem 283: 7599-7607.

22. Osta MA, Christophides GK, Vlachou D, Kafatos FC (2004) Innate immunity in the malaria vector Anopheles gambiae: comparative and functional genomics. J Exp Biol 207: 2551-2563.

23. Michel K, Kafatos FC (2005) Mosquito immunity against Plasmodium. Insect Biochem Mol Biol 35: 677-689.

24. Hultmark D (2003) Drosophila immunity: paths and patterns. Curr Opin Immunol 15: 12-19.

25. Jiang H, Kanost MR (2000) The clip-domain family of serine proteinases in arthropods. Insect Biochem Mol Biol 30: 95-105.

26. Adams MD, Celniker SE, Holt RA, Evans CA, Gocayne JD, et al. (2000) The genome sequence of Drosophila melanogaster. Science 287: 2185-2195.

27. Christophides GK, Zdobnov E, Barillas-Mury C, Birney E, Blandin S, et al. (2002) Immunity-related genes and gene families in Anopheles gambiae. Science 298: 159-165.

28. Waterhouse RM, Kriventseva EV, Meister S, Xi Z, Alvarez KS, et al. (2007) Evolutionary dynamics of immune-related genes and pathways in diseasevector mosquitoes. Science 316: 1738-1743.

\section{Author Contributions}

Conceived and designed the experiments: YL GW CA. Performed the experiments: YL DS FZ. Analyzed the data: YL DS FZ GW CA. Contributed reagents/materials/analysis tools: YL DS FZ. Wrote the paper: YL DS FZ GW CA.

29. Evans JD, Aronstein K, Chen YP, Hetru C, Imler JL, et al. (2006) Immune pathways and defence mechanisms in honey bees Apis mellifera. Insect Mol Biol 15: $645-656$

30. Zou Z, Evans JD, Lu Z, Zhao P, Williams M, et al. (2007) Comparative genomic analysis of the Tribolium immune system. Genome Biol 8: R177.

31. Tanaka H, Ishibashi J, Fujita K, Nakajima Y, Sagisaka A, et al. (2008) A genome-wide analysis of genes and gene families involved in innate immunity of Bombyx mori. Insect Biochem Mol Biol 38: 1087-1110.

32. Huang W, Marth G (2008) EagleView: a genome assembly viewer for nextgeneration sequencing technologies. Genome Res 18: 1538-1543.

33. Zhang J, Chiodini R, Badr A, Zhang G (2011) The impact of next-generation sequencing on genomics. J Genet Genomics 38: 95-109.

34. Pascual L, Jakubowska AK, Blanca JM, Canizares J, Ferre J, et al. (2012) The transcriptome of Spodoptera exigua larvae exposed to different types of microbes. Insect Biochem Mol Biol 42: 557-570.

35. Bao YY, Qu LY, Zhao D, Chen LB, Jin HY, et al. (2013) The genome- and transcriptome-wide analysis of innate immunity in the brown planthopper, Nilaparvata lugens. BMC Genomics 14: 160.

36. Vogel H, Altincicek B, Glockner G, Vilcinskas A (2011) A comprehensive transcriptome and immune-gene repertoire of the lepidopteran model host Galleria mellonella. BMC Genomics 12: 308.

37. Afidchao MM, Musters C, de Snoo GR (2013) Asian corn borer (ACB) and non-ACB pests in GM corn (Zea mays L.) in the Philippines. Pest Manag Sci 69: 792-801

38. Wagner BL, Lewis LC (2000) Colonization of corn, Zea mays, by the entomopathogenic fungus Beauveria bassiana. Appl Environ Microbiol 66: 34683473 .

39. Qu M, Liu T, Yang J, Yang Q (2011) The gene, expression pattern and subcellular localization of chitin synthase B from the insect Ostrinia furnacalis. Biochem Biophys Res Commun 404: 302-307.

40. Grabherr MG, Haas BJ, Yassour M, Levin JZ, Thompson DA, et al. (2011) Full length transcriptome assembly from RNA-Seq data: without a reference genome. Nat Biotechnol 29: 644-U130.

41. Pertea G, Huang X, Liang F, Antonescu V, Sultana R, et al. (2003) TIGR Gene Indices clustering tools (TGICL): a software system for fast clustering of large EST datasets. Bioinformatics 19: 651-652.

42. Altschul SF, Madden TL, Schaffer AA, ZhangJ, Zhang Z, et al. (1997) Gapped BLAST and PSI-BLAST: a new generation of protein database search programs. Nucleic Acids Res 25: 3389-3402.

43. Iseli C, Jongeneel CV, Bucher P (1999) ESTScan: a program for detecting, evaluating, and reconstructing potential coding regions in EST sequences. Proc Int Conf Intell Syst Mol Biol: 138-148.

44. Conesa A, Gotz S, Garcia-Gomez JM, Terol J, Talon M, et al. (2005) Blast2GO: a universal tool for annotation, visualization and analysis in functional genomics research. Bioinformatics 21: 3674-3676.

45. Gotz S, Garcia-Gomez JM, Terol J, Williams TD, Nagaraj SH, et al. (2008) High-throughput functional annotation and data mining with the Blast2GO suite. Nucleic Acids Res 36: 3420-3435.

46. Langmead B, Trapnell C, Pop M, Salzberg SL (2009) Ultrafast and memoryefficient alignment of short DNA sequences to the human genome. Genome Biol 10: R25

47. Mortazavi A, Williams BA, McCue K, Schaeffer L, Wold B (2008) Mapping and quantifying mammalian transcriptomes by RNA-Seq. Nat Methods 5: 621-628.

48. Audic S, Claverie JM (1997) The significance of digital gene expression profiles. Genome Res 7: 986-995.

49. Benjamini Y, Hochberg Y (1995) Controlling the false discovery rate: a practical and powerful approach to multiple testing. J Royal Stat Soci Series B: 289-300

50. Tamura K, Peterson D, Peterson N, Stecher G, Nei M, et al. (2011) MEGA5: molecular evolutionary genetics analysis using maximum likelihood, evolutionary distance, and maximum parsimony methods. Mol Biol Evol 28: 2731-2739.

51. Livak KJ, Schmittgen TD (2001) Analysis of relative gene expression data using real-time quantitative PCR and the 2 (-Delta Delta $\mathrm{C}(\mathrm{T})$ ) Method. Methods 25: $402-408$.

52. Hoffmann JA, Kafatos FC, Janeway CA, Ezekowitz RA (1999) Phylogenetic perspectives in innate immunity. Science 284: 1313-1318.

53. Yoshida H, Kinoshita K, Ashida M (1996) Purification of a peptidoglycan recognition protein from hemolymph of the silkworm, Bombyx mori.J Biol Chem 271: $13854-13860$.

54. Sumathipala N, Jiang H (2010) Involvement of Manduca sexta peptidoglycan recognition protein-1 in the recognition of bacteria and activation of prophenoloxidase system. Insect Biochem Mol Biol 40: 487-495.

55. Kurata S (2013) Peptidoglycan recognition proteins in Drosophila immunity. Dev Comp Immunol. doi: 10.1016/j.dci.2013.06.006. 
56. Werner T, Liu G, Kang D, Ekengren S, Steiner H, et al. (2000) A family of peptidoglycan recognition proteins in the fruit fly Drosophila melanogaster. Proc Natl Acad Sci U S A 97: 13772-13777.

57. Kim YS, Ryu JH, Han SJ, Choi KH, Nam KB, et al. (2000) Gram-negative bacteria-binding protein, a pattern recognition receptor for lipopolysaccharide and beta-1, 3-glucan that mediates the signaling for the induction of innate immune genes in Drosophila melanogaster cells. J Biol Chem 275: 32721-32727.

58. Ochiai M, Ashida M (1988) Purification of a beta-1, 3-glucan recognition protein in the prophenoloxidase activating system from hemolymph of the silkworm, Bombyx mori. J Biol Chem 263: 12056-12062.

59. Ma C, Kanost MR (2000) A beta 1, 3-glucan recognition protein from an insect, Manduca sexta, agglutinates microorganisms and activates the phenoloxidase cascade. J Biol Chem 275: 7505-7514.

60. Jiang H, Ma C, Lu ZQ, Kanost MR (2004) Beta-1, 3-glucan recognition protein-2 (betaGRP-2)from Manduca sexta;an acute-phase protein that binds beta-1,3-glucan and lipoteichoic acid to aggregate fungi and bacteria and stimulate prophenoloxidase activation. Insect Biochem Mol Biol 34: 89-100.

61. Ochiai M, Ashida M (2000) A pattern-recognition protein for beta-1, 3-glucan. The binding domain and the cDNA cloning of beta-1,3-glucan recognition protein from the silkworm, Bombyx mori. J Biol Chem 275: 4995-5002.

62. Takahasi K, Ochiai M, Horiuchi M, Kumeta H, Ogura K, et al. (2009) Solution structure of the silkworm betaGRP/GNBP3 N-terminal domain reveals the mechanism for beta-1, 3-glucan-specific recognition. Proc Natl Acad Sci U S A 106: 11679-11684.

63. Mishima Y, Quintin J, Aimanianda V, Kellenberger C, Coste F, et al. (2009) The N-terminal domain of Drosophila Gram-negative binding protein 3 (GNBP3) defines a novel family of fungal pattern recognition receptors. J Biol Chem 284: 28687-28697.

64. Yu XQ Kanost MR (2004) Immulectin-2, a pattern recognition receptor that stimulates hemocyte encapsulation and melanization in the tobacco hornworm, Manduca sexta. Dev Comp Immunol 28: 891-900.

65. Yu XQ, Kanost MR (2000) Immulectin-2, a lipopolysaccharide-specific lectin from an insect, Manduca sexta, is induced in response to gram-negative bacteria. J Biol Chem 275: 37373-37381.

66. Koizumi N, Imamura M, Kadotani T, Yaoi K, Iwahana H, et al. (1999) The lipopolysaccharide- binding protein participating in hemocyte nodule formation in the silkworm Bombyx mori is a novel member of the C-type lectin superfamily with two different tandem carbohydrate-recognition domains. FEBS Lett 443: 139-143.

67. Yu XQ Kanost MR (2003) Manduca sexta lipopolysaccharide-specific immulectin-2 protects larvae from bacterial infection. Dev Comp Immunol 27: 189-196.

68. Shin SW, Park DS, Kim SC, Park HY (2000) Two carbohydrate recognition domains of Hyphantria cunea lectin bind to bacterial lipopolysaccharides through O-specific chain. FEBS Lett 467: 70-74.

69. Leshko-Lindsay LA, Corces VG (1997) The role of selectins in Drosophila eye and bristle development. Development 124: 169-180.

70. Kanost MR, Jiang H, Wang Y, Yu XQ Ma C, et al. (2001) Hemolymph proteinases in immune responses of Manduca sexta. Adv Exp Med Biol 484: 319328.

71. Zou Z, Lopez DL, Kanost MR, Evans JD, Jiang H (2006) Comparative analysis of serine protease-related genes in the honey bee genome: possible involvement in embryonic development and innate immunity. Insect Mol Biol 15: 603-614.

72. Lee KY, Zhang R, Kim MS, Park JW, Park HY, et al. (2002) A zymogen form of masquerade-like serine proteinase homologue is cleaved during prophenoloxidase activation by $\mathrm{Ca}^{2+}$ in coleopteran and Tenebrio molitor larvae. Eur J Biochem 269: 4375-4383.

73. Yu XQ Jiang H, Wang Y, Kanost MR (2003) Nonproteolytic serine proteinase homologs are involved in prophenoloxidase activation in the tobacco hornworm, Manduca sexta. Insect Biochem Mol Biol 33: 197-208.

74. Kan H, Kim CH, Kwon HM, Park JW, Roh KB, et al. (2008) Molecular control of phenoloxidase- induced melanin synthesis in an insect. J Biol Chem 283: 25316-25323.

75. Ross J, Jiang H, Kanost MR, Wang Y (2003) Serine proteases and their homologs in the Drosophila melanogaster genome: an initial analysis of sequence conservation and phylogenetic relationships. Gene 304: 117-131.

76. Jang IH, Nam HJ, Lee WJ (2008) CLIP-domain serine proteases in Drosophila innate immunity. BMB reports 41: 102-107.

77. Zou Z, Picheng Z, Weng H, Mita K, Jiang H (2009) A comparative analysis of serpin genes in the silkworm genome. Genomics 93: 367-375.

78. Ji C, Wang Y, Guo X, Hartson S, Jiang H (2004) A pattern recognition serine proteinase triggers the prophenoloxidase activation cascade in the tobacco hornworm, Manduca sexta. J Biol Chem 279: 34101-34106.

79. Kanost MR (1999) Serine proteinase inhibitors in arthropod immunity. Dev Comp Immunol 23: 291-301.

80. Silverman GA, Whisstock JC, Bottomley SP, Huntington JA, Kaiserman D, et al. (2010) Serpins flex their muscle: I. Putting the clamps on proteolysis in diverse biological systems. J Biol Chem 285: 24299-24305.

81. Gettins PG (2002) Serpin structure, mechanism, and function. Chem Rev 102: 4751-4803.

82. Michel K, Budd A, Pinto S, Gibson TJ, Kafatos FC (2005) Anopheles gambiae SRPN2 facilitates midgut invasion by the malaria parasite Plasmodium berghei. EMBO reports 6: 891-897.
83. An C, Budd A, Kanost MR, Michel K (2011) Characterization of a regulatory unit that controls melanization and affects longevity of mosquitoes. Cell Mol Life Sci 68: 1929-1939.

84. Ligoxygakis P, Pelte N, Ji C, Leclerc V, Duvic B, et al. (2002) A serpin mutant links Toll activation to melanization in the host defence of Drosophila. EMBO J 21: $6330-6337$.

85. De Gregorio E, Han SJ, Lee WJ, Baek MJ, Osaki T, et al. (2002) An immuneresponsive Serpin regulates the melanization cascade in Drosophila. Dev Cell 3: $581-592$.

86. Zhu Y, Wang Y, Gorman MJ, Jiang H, Kanost MR (2003) Manduca sexta serpin-3 regulates prophenoloxidase activation in response to infection by inhibiting prophenoloxidase-activating proteinases. J Biol Chem 278: 46556-46564.

87. Tong Y, Jiang H, Kanost MR (2005) Identification of plasma proteases inhibited by Manduca sexta serpin- 4 and serpin- 5 and their association with components of the prophenol oxidase activation pathway. J Biol Chem 280: 14932-14942.

88. Jiang R, Kim EH, Gong JH, Kwon HM, Kim CH, et al. (2009) Three pairs of protease-serpin complexes cooperatively regulate the insect innate immune responses. J Biol Chem 284: 35652-35658.

89. Weber AN, Tauszig-Delamasure S, Hoffmann JA, Lelievre E, Gascan H, et al. (2003) Binding of the Drosophila cytokine Spatzle to Toll is direct and establishes signaling. Nat Immunol 4: 794-800.

90. Wang Y, Cheng T, Rayaprolu S, Zou Z, Xia Q et al. (2007) Proteolytic activation of pro-spatzle is required for the induced transcription of antimicrobial peptide genes in lepidopteran insects. Dev Comp Immunol 31: $1002-1012$.

91. An C, Jiang H, Kanost MR (2010) Proteolytic activation and function of the cytokine Spatzle in the innate immune response of a lepidopteran insect, Manduca sexta. FEBS J 277: 148-162.

92. DeLotto Y, DeLotto R (1998) Proteolytic processing of the Drosophila Spatzle protein by easter generates a dimeric NGF-like molecule with ventralising activity. Mech Dev 72: 141-148.

93. Michel T, Reichhart JM, Hoffmann JA, Royet J (2001) Drosophila Toll is activated by Gram-positive bacteria through a circulating peptidoglycan recognition protein. Nature 414: 756-759.

94. Rock FL, Hardiman G, Timans JC, Kastelein RA, Bazan JF (1998) A family of human receptors structurally related to Drosophila Toll. Proc Natl Acad Sci U S A 95: 588-593.

95. Xu Y, Tao X, Shen B, Horng T, Medzhitov R, et al. (2000) Structural basis for signal transduction by the Toll/interleukin-1 receptor domains. Nature 408: $111-115$

96. Cheng TC, Zhang YL, Liu C, Xu PZ, Gao ZH, et al. (2008) Identification and analysis of Toll-related genes in the domesticated silkworm, Bombyx mori. Dev Comp Immunol 32: 464-475.

97. Wang L, Ligoxygakis P (2006) Pathogen recognition and signalling in the Drosophila innate immune response. Immunobiology 211: 251-261.

98. Hoffmann JA, Reichhart JM (2002) Drosophila innate immunity: an evolutionary perspective. Nat Immunol 3: 121-126.

99. Zhou R, Silverman N, Hong M, Liao DS, Chung Y, et al. (2005) The role of ubiquitination in Drosophila innate immunity. J Biol Chem 280: 34048-34055.

100. Kawabata T, Yasuhara Y, Ochiai M, Matsuura S, Ashida M (1995) Molecular cloning of insect pro-phenol oxidase: a copper-containing protein homologous to arthropod hemocyanin. Proc Natl Acad Sci U S A 92: 7774-7778.

101. Wang Y, Jiang H (2004) Prophenoloxidase (proPO) activation in Manduca sexta: an analysis of molecular interactions among proPO, proPO-activating proteinase-3, and a cofactor. Insect Biochem Mol Biol 34: 731-742.

102. Feng C, Song O Lü W, Lu J (2008) Purification and characterization of hemolymph prophenoloxidase from Ostrinia furnacalis (Lepidoptera: Pyralidae) larvae. Comp Biochem Physiol B Biochem Mol Biol 151: 139-146.

103. Yasuhara Y, Koizumi Y, Katagiri C, Ashida M (1995) Reexamination of properties of prophenoloxidase isolated from larval hemolymph of the silkworm Bombyx mori. Arch Biochem Biophys 320: 14-23.

104. Andreu D, Rivas L (1998) Animal antimicrobial peptides: an overview. Biopolymers 47: 415-433.

105. Brogden KA (2005) Antimicrobial peptides: pore formers or metabolic inhibitors in bacteria? Nat Rev Microbiol 3: 238-250.

106. Bachali S, Jager M, Hassanin A, Schoentgen F, Jolles P, et al. (2002) Phylogenetic analysis of invertebrate lysozymes and the evolution of lysozyme function. J Mol Evol 54: 652-664.

107. Jimenez-Cantizano RM, Infante C, Martin-Antonio B, Ponce M, Hachero I, et al. (2008) Molecular characterization, phylogeny, and expression of c-type and g-type lysozymes in brill (Scophthalmus rhombus). Fish Shellfish Immunol 25: 57-65.

108. Gandhe AS, Janardhan G, Nagaraju J (2007) Immune upregulation of novel antibacterial proteins from silkmoths (Lepidoptera) that resemble lysozymes but lack muramidase activity. Insect Biochem Mol Biol 37: 655-666.

109. Steiner H, Hultmark D, Engstrom A, Bennich H, Boman HG (1981) Sequence and specificity of two antibacterial proteins involved in insect immunity. Nature 292: 246-248.

110. Bulet P, Stocklin R, Menin L (2004) Anti-microbial peptides: from invertebrates to vertebrates. Immunol Rev 198: 169-184.

111. Knight JA (2000) Review: Free radicals, antioxidants, and the immune system. Ann Clin Lab Sci 30: 145-158. 